\title{
COMPACT SELF-DUAL MANIFOLDS WITH POSITIVE SCALAR CURVATURE
}

\author{
Y. SUN POON
}

\section{Introduction}

This article is the author's exercise in attempting to understand Hitchin's work on the classification of compact Kählerlian twistor space [21]. The motivation is from Langer's question on whether there is a self-dual metric on the connected sum of the complex projective planes.

A classification theorem in Riemannian geometry is the following [15], [22]: If $X$ is a compact self-dual Einstein manifold with positive scalar curvature, then $X$ is isometric to the Euclidean 4-sphere $S^{4}$ or the complex projective plane $\mathbf{C} \mathbf{P}^{2}$ with the Fubini-Study metric.

We ask whether there is any self-dual metric with positive scalar curvature on a compact simply connected manifold which is not conformally equivalent to these standard metrics on $S^{4}$ or $\mathbf{C P}^{2}$. Our answer is contained in the following two theorems:

Theorem A. Suppose that $X$ is a compact simply connected self-dual manifold with positive scalar curvature. If the signature of $X$ is equal to zero or one, then $X$ is conformally equivalent to the Euclidean 4-sphere or the projective plane with Fubini-Study metric.

Theorem B. (i) There exists a one-parameter family of self-dual conformal classes on $\mathbf{C P}^{2} \# \mathbf{C} \mathbf{P}^{2}$, the connected sum of two complex projective planes with usual orientation.

(ii) Each of these conformal classes contains a metric with positive scalar curvature.

(iii) Each of these conformal classes has a two-dimensional torus as the identity component of the group of conformal transformation.

Received January 2, 1986. The author is partially supported by a grant of the National Research Foundation. 
(iv) The twistor space of each of these conformal classes is a small resolution of the intersection of two quadrics in $\mathbf{C P}^{5}$ given by

$$
\begin{aligned}
& Q_{0}=\left\{z \in \mathbf{C P}^{5}: 2 z_{0}^{2}+2 z_{1}^{2}+\lambda z_{2}^{2}+\frac{3}{2} z_{3}^{2}+z_{4}^{2}+z_{5}^{2}=0\right\} \\
& Q_{\infty}=\left\{z \in \mathbf{C P}^{5}: z_{0}^{2}+z_{1}^{2}+z_{2}^{2}+z_{3}^{2}+z_{4}^{2}+z_{5}^{2}=0\right\}
\end{aligned}
$$

for a real number $\lambda$ such that $2>\lambda>\frac{3}{2}$.

(v) Let the conformal class associated to the number $\lambda$ be denoted by $C_{\lambda}$.

Suppose that $X$ is a compact simply connected self-dual manifold with positive scalar curvature. If the signature of $X$ is equal to two, then $X$ is conformally equivalent to $\mathbf{C P}^{2} \# \mathbf{C P}^{2}$ with a $C_{\lambda}$.

A useful corollary of Theorem A is that the conformal class of the FubiniStudy metric is the unique self-dual conformal class on the projective plane admitting metrics with positive scalar curvature. However, the proof of Theorem B will occupy most of our time.

It is well known that self-duality on a manifold is a conformally invariant property, and that the conformal structure is uniquely determined by the holomorphic structure of the twistor space [3], [20], [28]. Therefore, our basic method is to exploit Hitchin's techniques in [21] to look for the candidates of twistor spaces and then use the Penrose transform to construct self-dual conformal classes on $\mathbf{C P}^{2} \# \mathbf{C} \mathbf{P}^{2}$. As the second step is standard, to prove Theorem B our major task is to take the first step. In [21], Hitchin showed that the only compact Kählerian twistor spaces are $\mathbf{C P}^{3}$ and the flag manifold of lines in $\mathbf{C P}^{2}$. During the course of the proof, he found that if a self-dual manifold has Kählerian twistor space and has signature 2, then the twistor space is identified with the complete intersection of the two quadrics. But this identification given by the Kählerian assumption is not compatible with the topological assumption that $\tau=2$. Bearing this observation in mind, our objective is a singular model of a twistor space. The singularity is related to and hopefully is determined by the topology of the manifold $\mathbf{C P}^{2} \# \mathbf{C P}^{2}$.

In the first three sections, we shall always suppose that we are given a compact simply-connected self-dual manifold $X$ with positive scalar curvature. In $\$ 1$, basic definitions will be given and useful information, e.g. a topological classification and a vanishing theorem of Hitchin, will be collected. In $\S 2$, we imitate Hitchin's method of studying the linear system $\left|-\frac{1}{2} K\right|$ when the signature of $X$ is not bigger than two. As many technical observations in this section are simple refinements of Hitchin's argument in [21], we shall not give every detail. Instead, we shall concentrate on proving Theorem A. Theorem A 
is true because when the signature is small, the system $\left|-\frac{1}{2} K\right|$ provides us enough degree of freedom to show that its associated map $\Phi$ is an embedding. This is true even if one replaces the assumption of simple-connectivity by that of vanishing first betti number.

In $\S 3$, we study the case when the signature is equal to two. From the work in $\S 2$, we shall see that the obstruction for the map $\Phi$ to be an embedding is essentially from a collection of curves on which the bundle $\mathbf{K}^{-1 / 2}$ is trivial. The image of these curves are singular points on the projective model of the twistor space. We shall introduce four holomorphic line bundles on the twistor space to locate these curves. These bundles are determined by the topology of $X$ and in fact determine the singularity of $\Phi(Z)$. Their interrelation is so rigid that they determine the algebraic type of the image of the twistor space. The key observation is Remark 3.3.

After determining the only possible singular model of the twistor space, we change our viewpoint in $\$ 4$. Our aim is to use this model to construct a self-dual conformal class on $\mathbf{C P}^{2} \# \mathbf{C} \mathbf{P}^{2}$. Away from the singularity, it is easy to use the Penrose programme to work out the construction on an open set especially when we have Hurtubise's work as a reference [24]. A metric in the self-dual conformal class will be expressed in terms of coordinate functions. It has apparent singularity. But with the picture in $\$ 3$ in mind, we are able to resolve the coordinate singularity. Then in $\$ 5$, after proving several general observations on the sign of the scalar curvature of a self-dual metric, we use the solution of Yamabe's problem to show that we have a self-dual conformal class on $\mathbf{C P}^{2} \# \mathbf{C P}^{2}$ in which there is a metric with positive scalar curvature. Theorem $B$ is then the collection of Theorems 5.4, 5.5, 5.6, and 3.5.

In §7. Appendix, we shall give an explicit construction of the moduli space of self-dual $S O(3)$-connections over the connected-sum of two complex projective planes.

Our basic reference is Atiyah, Hitchin and Singer [3]. Concerning the holomorphic geometry of twistor spaces, readers are referred to [21]. The references for algebraic geometry are [16] and [23].

For convenience, when the bold capital letters denote holomorphic line bundles, the usual italic capital letters will denote the corresponding divisor classes. For example $\mathbf{K}^{-1}$ is the anticanonical bundle and $-K$ is the anticanonical class. $\mathbf{H}$ will always denote the hyperplane bundle on a projective space or a nonsingular rational curve.

Acknowledgment. I am most grateful to N. J. Hitchin for his teaching and encouragement. I would also like to thank the Croucher Foundation of Hong Kong for financial support when I was in Oxford from 1981 to 1985. 


\section{Preliminaries}

Let $X$ be an oriented Riemannian manifold such that (i) it is compact, connected and simply-connected, and (ii) it is self-dual and has positive scalar curvature.

To determine the topological type of this manifold, we can write down the Weitzenböch formula of the exterior derivative restricted to the anti-self-dual 2-forms. Using this formula, we can apply a Bochner type argument involving the above geometrical assumption to show that the intersection form on the second cohomology is positive definite. After Freedman and Donaldson's work [11], [14], it is now well known that the manifold $X$ has to be homeomorphic to the 4-sphere or the connected sum of the complex projective planes with natural orientation. Therefore, the homeomorphic type of $X$ is uniquely determined by its signature $\tau$. The Euler characteristic $\chi$ of $X$ is given by $\chi=2+\tau$.

When $\tau=0$, the conformal structure of $X$ can easily be recognized because, in this case, $X$ is necessarily conformally flat. According to Kuiper [25], a compact, simply-connected, conformally flat 4-manifold is conformally equivalent to the Euclidean 4-sphere. To determine the conformal structure on $X$ when $\tau=1$ and $\tau=2$, we shall study the twistor space over $X$.

By definition, the twistor space $Z$ over an oriented Riemannian 4dimensional manifold is the total space of the projective spin $-\frac{1}{2}$-bundle $\mathbf{P}\left(\mathbf{V}_{-}\right)$. The fibration is called the twistor fibration. An almost complex structure on the twistor space is defined as follows: The space of 1 -forms is split into vertical and horizontal parts by the induced connection. The vertical $(1,0)$-form at a point $z$ in the fiber $\mathbf{P}\left(\mathbf{V}_{-}\right)_{x}$ is chosen to be the $(1,0)$-form of the fiber $\mathbf{C P}^{1}$ at $z$. On the horizontal part, the space of $(1,0)$-forms is chosen to be $\left(\mathbf{V}_{+}\right)_{x} \otimes v$, where $\mathbf{V}_{+}$is the spin $+\frac{1}{2}$-bundle and $v$ is any nonzero $-\frac{1}{2}$ spinor defining the point $z$ in $\mathbf{P}\left(\mathbf{V}_{-}\right)_{x}$. Here we are using the isomorphism between the complexified bundle of 1 -forms over $X$ and the tensor product of $+\frac{1}{2}$ spin and $-\frac{1}{2}$ spin bundles. It is known [3] that this almost complex structure depends only on the conformal class of the metric and when the metric is self-dual, this almost complex structure is integrable. Then in our case, the twistor space $Z$ is a compact complex manifold. Note that the fibers are holomorphic rational curves with normal bundle $\mathbf{H} \oplus \mathbf{H}$.

On the twistor space, there is a canonically defined bundle: If we remove the zero vector of the spin $-\frac{1}{2}$ representation space $V_{-}$of the Lie group $S U(2) \times$ $S U(2)$, and identify a vector $v$ to $-v$, then we have a manifold $\left(V_{-} \backslash 0\right) / \mathbf{Z}_{2}$ on which $S O(4)$ acts. This action defines an associated fiber bundle over $X$. This bundle is pulled back onto the twistor space to be a principal $\mathbf{C}^{*}$-bundle. The usual complex multiplication defines an associated line bundle $\mathbf{K}^{1 / 2}$ which is a 
holomorphic line bundle such that $\left(\mathbf{K}^{1 / 2}\right)^{2}=\mathbf{K}$, the canonical bundle on the complex manifold $Z$. From this tautological construction, one can check that $\mathbf{K}^{1 / 2}$ is restricted to be $\mathbf{H}^{-2}$ on each fiber and that the second Stiefel-Whitney class of the underlying $S O(2)$-bundle of $\mathbf{K}^{1 / 2}$ is precisely the second StiefelWhitney class of the manifold $X: w_{2}\left(\mathbf{K}^{1 / 2}\right)=w_{2}(X)$. This equality is understood through the Leray-Hirsch Theorem [32].

There is also a canonically defined structure on $Z$ : The $-\frac{1}{2}$ spin space $V_{-}$is a quaternionic representation space of $S U(2) \times S U(2)$. The quaternionic structure induces a fixed point free antiholomorphic involution on the twistor space. Following Atiyah, this involution is called a real structure and we may ask whether a complex surface in $Z$ is real or not and talk of the conjugate divisor of a divisor, and so on. For example, as the fibers are invariant under the real structure, then these copies of complex projective lines are called real twistor lines. One may also spell out the definitions to see that $\mathbf{K}^{1 / 2}$ is a real bundle.

A useful aspect of the above construction is that the complex structure of the twistor space determines the self-dual conformal structure on $X$. Explicitly, we can consider the twistor space as a complex 3-fold fibered by a real family of complex projective lines, each with normal bundle $\mathbf{H} \oplus \mathbf{H}$. From a theorem of Kodaira, one knows that each of these lines belongs to a complete local 4-complex parameter family $X^{c}$ and that there is a canonical isomorphism between the space of holomorphic sections of the normal bundle of a fiber $L_{x}$ and the tangent space at the parameter $x$. Then the quadratic cone of normal sections that vanish at some point of $L_{x}$ gives $X^{c}$ a complex conformal structure. This is the infinitesimal analogue of a family of lines intersecting at a point. Restricted to the parameter space $X$ of real lines, the complex structure gives a positive definite conformal class. This conformal class is exactly the one we use to construct the complex structure on the twistor space. Therefore, we can determine the conformal structure on $X$ by determining the holomorphic structure of the twistor space. This correspondence between self-dual conformal geometry on $X$ and the structures on twistor space is called the Penrose correspondence. Now let $c_{j}$ be the $j$ th Chern class on $Z$. Making use of the fact that $Z$ is an $S^{2}$-bundle over $X$, one can prove [21] that

$$
c_{1}^{3}=16(4-\tau), \quad c_{1} c_{2}=24, \quad c_{3}=4+2 \tau
$$

Here we see that the Todd genus of $Z$ is equal to 1 .

Note that the bundle $\mathbf{K}^{n / 2}$ is restricted to be a bundle of negative degree on each real twistor line when $n$ is a natural number. Therefore, $\mathbf{K}^{n / 2}$ does not have nontrivial global holomorphic sections. In particular, one can use Serre 
duality to check that $H^{3}(Z, \mathcal{O})$ vanishes. As the Todd genus of $Z$ is equal to 1 , then

$$
h^{1}(Z, \mathcal{O})=-1+h^{0}(Z, \mathcal{O})+h^{2}(Z, \mathcal{O})=h^{2}(Z, \mathcal{O})=h^{1}(Z, \mathcal{O}(\mathbf{K}))
$$

As the scalar curvature of the self-dual metric on $X$ is positive, Hitchin's vanishing theorem [20] shows that $h^{1}(Z, \mathcal{O}(\mathbf{K}))=0$. Therefore, $h^{1}(Z, \mathcal{O})$ and $h^{2}(Z, \mathcal{O})$ are both equal to zero. It follows that every second integral cohomology class is the first Chern class of a unique holomorphic line bundle on $Z$.

Let $\alpha_{1}, \cdots, \alpha_{\tau}$ be the generators of $H^{2}(X, \mathbf{Z})$ such that $\alpha_{i} \alpha_{j}=\delta_{i j}$. They are pulled back to the twistor space. Then we have the following cohomology class:

$$
\frac{1}{4} c_{1}+\frac{1}{2} \sum_{j=1}^{\tau} \varepsilon_{j} \alpha_{j}, \quad \varepsilon_{j}= \pm 1
$$

Neither $\frac{1}{4} c_{1}$ nor $\frac{1}{2} \sum_{j=1}^{\tau} \varepsilon_{j} \alpha_{j}$ is integral class, but the equality $w_{2}\left(\mathbf{K}^{1 / 2}\right)=w_{2}(X)$ implies that $\frac{1}{4} c_{1}+\frac{1}{2} \sum \varepsilon_{j} \alpha_{j}$ is an integral class and hence is the first Chern class of a unique line bundle $\mathbf{D}_{\varepsilon_{1} \varepsilon_{2}} \cdots \varepsilon_{\varepsilon^{*}}$.

Theorem (Hitchin [20]). (i) $h^{0}\left(Z, \mathcal{O}\left(\mathbf{K}^{n / 2}\right)\right)=0$ for all $n \geqslant 1$,

(ii) $h^{1}\left(Z, \mathcal{O}\left(\mathbf{K}^{n / 2}\right)\right)=0$ for all $n \geqslant 1$,

(iii) $h^{2}\left(Z, \mathcal{O}\left(\mathbf{D}_{\varepsilon_{1}} \cdots \varepsilon_{\tau}\right)\right)=0$.

We have indicated the proof of (i). For the proof of (ii) and (iii), the reader is referred to Corollary (3.8) in [20]. We shall use this set of vanishing theorems so often that we may not mention them when they are in use.

\section{The linear system $\left|-\frac{1}{2} K\right|$, when $\tau \leqslant 2$}

Given any holomorphic line bundle $\mathbf{D}$ on the twistor space with first Chern class $d$, its Euler characteristic $\chi(Z, \mathcal{O}(\mathbf{D}))$ is defined to be

$$
\chi(Z, \mathcal{O}(\mathbf{D}))=\sum_{j}(-1)^{j} h^{j}(Z, \mathcal{O}(\mathbf{D})) .
$$

According to the Riemann-Roch formula,

$$
\chi(Z, \mathcal{O}(\mathbf{D}))=\frac{1}{6} d^{3}+\frac{1}{4} d^{2} c_{1}+\frac{1}{12} d\left(c_{1}^{2}+c_{2}\right)+\frac{1}{24} c_{1} c_{2} .
$$

From this formula and Hitchin's vanishing theorem, we deduce that

$$
h^{0}\left(Z, \mathcal{O}\left(\mathbf{K}^{-1 / 2}\right)\right) \geqslant 2(5-\tau) \text {. }
$$

Under our assumption that $\tau \leqslant 2$, the bundle $\mathbf{K}^{-1 / 2}$ has nontrivial sections. We shall use $-\frac{1}{2} K$ to denote the divisor class corresponding to $\mathbf{K}^{-1 / 2}$ and $\left|-\frac{1}{2} K\right|$ the complete linear system of effective divisors linearly equivalent to $-\frac{1}{2} K$. The associated map of $\left|-\frac{1}{2} K\right|$ is denoted by $\Phi$. When $s_{0}, \cdots, s_{n}$ is a base 
of the vector space $H^{0}\left(Z, \mathcal{O}\left(\mathbf{K}^{-1 / 2}\right)\right), \Phi$ is a map from $Z$ into $\mathbf{C P}^{n}$ defined to be $\Phi(z)=\left[s_{0}(z): \cdots: s_{n}(z)\right]$ for any point $z$ in $Z$.

Lemma 2.1. If $S$ is an effective divisor in the twistor space, then $S \cdot L>0$ for any real twistor line.

Proof. As $Z \rightarrow X$ is a fibration with real twistor lines as fibers, $S$ must intersect a real twistor line $L$. Therefore $S \cdot L$ could be nonpositive only if $L$ is contained in $S$. It implies that a section $s$ of the divisor bundle $\mathbf{S}$ of $S$ vanishes along the twistor line $L$. By analyticity, it can vanish along $L$ up to a finite order $k$. In other words, when $\mathfrak{\Im}$ is the ideal sheaf of $L$ and $\mathbf{N}^{*}$ the dual of the normal bundle of $L$, then $s$ is a global section of $\mathfrak{J}^{k} \otimes \mathcal{O}(\mathbf{S})$ but is not a section of $\mathfrak{\Im}^{k+1} \otimes \mathcal{O}(\mathbf{S})$. Then the $k$ th derivative of $s$ is a well-defined nonzero section of the bundle $\mathbf{S} \otimes S^{k}\left(\mathbf{N}^{*}\right)$ on $L$, where $S^{k}\left(\mathbf{N}^{*}\right)$ is the $k$ th symmetric power of $\mathbf{N}^{*}$. As $\mathbf{N}=\mathbf{H} \oplus \mathbf{H}, \mathbf{S} \otimes S^{k}\left(\mathbf{N}^{*}\right)$ can have nontrivial section only if $\mathbf{S}$ has positive degree on $L$. That is $S \cdot L>0$.

Since $\mathbf{K}^{-1 / 2}$ is a real bundle, $H^{0}\left(Z, \mathcal{O}\left(\mathbf{K}^{-1 / 2}\right)\right)$ is a real vector space in the sense of [1]. Let $V \subseteq H^{0}\left(Z, \mathcal{O}\left(K^{-1 / 2}\right)\right)$ be a real vector subspace such that the system $|V|$ has dimension greater than 1 . If $|V|$ has fixed component, a generic real element $D$ in $|V|$ has the form $D=F+E$, where $F$ is the fixed part of $|V|$. If $F$ is considered to be the intersection of all elements in $|V|$, it is obvious that $F$ is a real effective divisor on $Z$. As $D$ is real, $E$ is also real and effective. Using the last lemma and reality, we have $F \cdot L \geqslant 2$ and $E \cdot L \geqslant 2$. This is impossible because $-\frac{1}{2} K \cdot L=2$. Therefore, any nontrivial real subsystem $|V|$ of $\left|-\frac{1}{2} K\right|$ does not have fixed component. Moreover, there are the two following general versions of Bertini's theorem [33].

Theorem 2.2. Let $|V|$ be a linear system with dimension $n$ on a compact complex manifold $Z, \Phi_{V}: Z \rightarrow \mathbf{C P}^{n}$ the associated meromorphic map. If $|V|$ does not have fixed components and $\operatorname{dim} \Phi_{V}(Z) \geqslant 2$, then a generic member of $|V|$ is an irreducible subvariety of $Z$.

Theorem 2.3. A generic element of a linear system $|V|$ on $Z$ cannot have singular points that are not base points of the system.

With these two theorems and the fact that the nontrivial real subsystem of $\left|-\frac{1}{2} K\right|$ has no fixed component, one can directly adopt Hitchin's argument in (ii) and (iii) of Proposition (4.3) in [21] to show the following.

Lemma 2.4. If $V \subseteq H^{0}\left(Z, \mathcal{O}\left(\mathrm{K}^{-1 / 2}\right)\right)$ is a real vector subspace such that $\operatorname{dim}|V| \geqslant 3$, a generic real element in $|V|$ is irreducible nonsingular.

To describe an irreducible nonsingular element in $\left|-\frac{1}{2} K\right|$ we have

Lemma 2.5. Suppose that $S$ in $\left|-\frac{1}{2} K\right|$ is a real nonsingular irreducible element; then it is the blowing up of a rational ruled surface $2 \tau$-times. This surface can also be blown down to the projective plane. The fixed component of its 
anticanonical system is a sum of irreducible components of reducible fibers of the ruling.

Proof. First of all, we claim that the surface $S$ must contain a real twistor line. To see this, let $s$ be a real section of $\mathbf{K}^{-1 / 2}$ defining $S$. Given any $x \in X$, let $Z_{x}$ be the fiber of the twistor space at $x$; then $s$ is restricted to be $s_{x}$ in $H^{0}\left(Z_{x}, \mathcal{O}\left(\mathbf{K}^{-1 / 2}\right)\right)$. Recall that $Z_{x}$ is the projective spinors $\mathbf{P}\left(\mathbf{V}_{-}\right)_{x}$ and $\mathbf{K}^{-1 / 2}$ is restricted to be $\mathbf{H}^{2}$ on $\mathbf{P}\left(\mathbf{V}_{-}\right)_{x}$. Therefore, $s_{x}$ is a homogeneous polynomial of degree 2 with variables in $\mathbf{V}_{-x}$. Using the symplectic structure to identify $\mathbf{V}_{-x}$ and $\mathbf{V}_{-x}^{*}$, we regard $s_{x}$ as a vector in the symmetric tensor product $\mathbf{V}_{-x}^{2}$. As the bundle $\mathbf{V}_{-}^{2}$ is isomorphic to the complexified bundle of anti-self-dual 2-forms on $X$ and $s_{x}$ is real, then the assignment $x \mapsto s_{x}$ defines a section of anti-self-dual 2-forms. If $s$ does not vanish identically on any real twistor line, then each $s_{x}$ is nonzero. After the length of each $s_{x}$ is normalized, we use the metric on $X$ to identify 2-forms and skew-adjoint endomorphisms on tangent space. Then a unit anti-self-dual 2-form at a point on $X$ becomes an almost complex structure on the vector space $T_{x} X$. Its canonical orientation is opposite to the original one. Then our section $x \mapsto s_{x}$ is an almost complex structure on. $X$. If $c_{1}^{\prime}$ and $c_{2}^{\prime}$ denote the Chern classes of this almost complex structure, then $\chi=c_{2}^{\prime}$ and $-\tau=\frac{1}{3}\left(c_{1}^{\prime 2}-2 c_{2}^{\prime}\right)$. Moreover, the index theorem [4] gives that $\chi\left(T^{1.0} X\right)=\frac{1}{12}\left(c_{1}^{\prime 2}+c_{2}^{\prime}\right)$ is an integer. It follows that $\frac{1}{4}(\chi-\tau)$ should be an integer. But $\chi-\tau=2$. Therefore the section $s$ must vanish on some real twistor line $L$.

Using the adjunction formula on the twistor space, one deduces that the canonical bundle $\mathbf{K}_{S}$ of the surface $S$ is the restriction of $\mathbf{K}^{1 / 2}$. As the real twistor line $L$ is a rational curve such that $-\frac{1}{2} K \cdot L=2$, one can apply the adjunction formula on $S$ to show that $L^{2}=0$. On the other hand, the induced long exact cohomology sequence of the short exact sequence

$$
0 \rightarrow \mathcal{O}\left(\mathbf{K}^{1 / 2}\right) \rightarrow \mathcal{O}_{Z} \rightarrow \mathcal{O}_{S} \rightarrow 0
$$

shows that $h^{1}(S, \mathcal{O})=0$. When $L$ is considered as a divisor on the surface $S$, then $\operatorname{dim}|L|=1$. From Noether's lemma [16], we then know that $S$ is the blowing up of a rational ruled surface with $L$ as a nonsingular fiber. Actually, we have to blow up $2 \tau$-many times because

$$
c_{1}^{2}(S)=\left(-\frac{1}{2} K\right)\left(-\frac{1}{2} K\right)^{2}=8-2 \tau .
$$

Suppose that $F$ is the fixed component of $\left|-K_{S}\right|$, this is a real effective divisor on $S$. If $D$ is a real element in $\left|-K_{S}\right|$, then $D=F+E$, where $E$ is a real effective divisor. As we can use an exact sequence argument on the twistor 
space to check that $\operatorname{dim}\left|-K_{S}\right| \geqslant 8-2 \tau \geqslant 4$, we can always find a $D$ such that the corresponding movable part $E$ has the property that $E \cdot L>0$. By reality, we actually have $E \cdot L \geqslant 2$. Yet

$$
D \cdot L=-K_{S} \cdot L=-\frac{1}{2} K \cdot L=2,
$$

therefore $F \cdot L=0$. Since an irreducible effective divisor can have intersection number 0 with a generic fiber of the ruling only when it is an irreducible fiber or an irreducible component of a reducible fiber, and a generic fiber can always deform to another generic fiber, the components of $F$ can only be irreducible components of reducible fibers.

From the above observation, we see that the proper transform $\hat{E}_{\infty}$ of the infinity section of the minimal ruled model of $S$ is not in the fixed component of $\left|-K_{S}\right|$; then $-K_{S} \cdot \hat{E}_{\infty} \geqslant 0$. By the adjunction formula, we have $\hat{E}_{\infty}^{2} \geqslant-2$. Now it is obvious that $S$ can be obtained by blowing up $\mathbf{C P}^{2} 2 \tau+1$ times.

Proposition 2.6. When $\tau \leqslant 2$, the linear system $\left|-\frac{1}{2} K\right|$ has no fixed points.

Proof. We have seen the reason why $\left|-\frac{1}{2} K\right|$ has no fixed component. Suppose that $C$ is an irreducible curve in the twistor space such that $-\frac{1}{2} K \cdot C$ $<0$, then it is in the base locus of $\left|-\frac{1}{2} K\right|$. Let $L$ be any real twistor line passing through the curve $C$ at a point $w$. Let $\bar{w}$ be the conjugate point. Then $\bar{w}$ is also in the base locus of $\left|-\frac{1}{2} K\right|$. Picking up any other point $z$ on $L$, we use $\left|-\frac{1}{2} K\right|_{z}$ to denote the elements in $\left|-\frac{1}{2} K\right|$ containing the point $z$. Since every element in $\left|-\frac{1}{2} K\right|_{z}$ contains three points on $L$, namely $w, \bar{w}$ and $z$, while $-\frac{1}{2} K \cdot L=2$, every element in $\left|-\frac{1}{2} K\right|_{z}$ contains $L$. That is $\left|-\frac{1}{2} K\right|_{z}=\left|-\frac{1}{2} K\right|_{L}$. Therefore, $\operatorname{dim}\left|-\frac{1}{2} K\right|_{L} \geqslant 8-2 \tau \geqslant 4$. According to the last two lemmas, the generic real element $S$ in $\left|-\frac{1}{2} K\right|_{L}$ is a rational surface, such that the fixed component of $\left|-K_{S}\right|$ is in the reducible fibers. Now $-\frac{1}{2} K \cdot C<0$ implies that the curve $C$ is in the fixed component of $\left|-K_{S}\right|$. But by construction, this curve intersects a generic fiber on $S$, namely, the real twistor line $L$. This is a contradiction. Therefore any irreducible curve $C$ on the twistor space has the property that $-\frac{1}{2} K \cdot C \geqslant 0$. It follows that for any curve $C$ on any real nonsingular irreducible element $S$ in $\left|-\frac{1}{2} K\right|,-K_{S} \cdot C \geqslant 0$. In view of Lemma 2.5, we know that $S$ is the blowing up of $\mathbf{C P}^{2} 2 \tau+1$-times so that there is no nonsingular rational curve with self-intersection less than or equal to -3 . This surface is usually called a (degenerate) Del Pezzo surface [9], [29]. As a particular case of Demazure's work [9], $\left|-K_{S}\right|$ is proved to have no base point and a generic element in $\left|-K_{S}\right|$ is irreducible. As one can see, from the exact sequence

$$
0 \rightarrow \mathcal{O} \rightarrow \mathcal{O}\left(\mathbf{K}^{-1 / 2}\right) \rightarrow \mathcal{O}_{S}\left(\mathbf{K}_{S}^{-1}\right) \rightarrow 0
$$


that the base locus of $\left|-\frac{1}{2} K\right|$ on $Z$ is precisely that of $\left|-K_{S}\right|$ on $S,\left|-\frac{1}{2} K\right|$ does not have base point.

Theorem 2.7. A compact connected simply connected manifold with $\tau=1$ and a self-dual metric with positive scalar curvature is conformally equivalent to the projective plane with Fubini-Study metric.

Proof. According to Hitchin [21], if the twistor space of a compact selfdual manifold is Kählerian, the manifold is conformally equivalent to the sphere or projective plane with standard metrics. Therefore, to prove the theorem, we only have to prove that the associated map $\Phi$ is an embedding.

From the last proposition, we see that the map $\Phi$ is holomorphic. Under the assumption that $\tau=1$, we actually have a lot more degree of freedom to refine the argument in the proof to check that $-\frac{1}{2} K \cdot C>0$ for any irreducible curve $C$ in the twistor space. Then any nonsingular irreducible real element $S$ in $\left|-\frac{1}{2} K\right|$ is given by blowing up $\mathbf{C P}^{2}$ at three general points. As the map $\Phi$ is restricted to be the associated map of the anticanonical system on $S$ and this map on any such $S$ is an embedding, one can easily see that the map $\Phi$ is one-to-one.

To prove that $\Phi$ is an embedding, we pick up any point $z$ in the twistor space. Through this point, there is a unique real twistor line $L$. A generic real element of $\left|-\frac{1}{2} K\right|$ containing $z$ is the blowing up of three general points on $\mathbf{C P}^{2}$. This surface intersects the curve $L$ transversely at two points. On the other hand, one can apply Lemma 2.4 on the system $\left|-\frac{1}{2} K\right|_{L}$ to see that there is also a nonsingular irreducible real element in $\left|-\frac{1}{2} K\right|$ containing $L$. This surface is also the blowing up of $\mathbf{C P}^{2}$ at three general points. As the restrictions of $\Phi$ to both surfaces are embedding and $\Phi$ is one-to-one, the differential of $\Phi$ at $z$ has full rank. Therefore, $\Phi$ is an embedding.

Corollary 2.8. The conformal class of the Fubini-Study metric is the unique self-dual conformal containing a metric with positive scalar curvature on $\mathbf{C P}^{2}$.

Remark 2.9. When $\tau=0$, Lemma 2.5 implies that a generic real element in $\left|-\frac{1}{2} K\right|$ is the blowing up of $\mathbf{C P}^{2}$ at a single point. Therefore, one can easily prove that the map $\Phi$ is an embedding and hence $X$ is conformally equivalent to the Euclidean sphere. A careful reader may have realized that the dimension of $\left|-\frac{1}{2} K\right|$ is determined by the geometric property of the manifold and its Betti numbers $b_{j}$. When we study the system of $\left|-\frac{1}{2} K\right|$ and its elements, we only make use of the consequence of the simple-connectivity that $b_{1}=0$ rather than the strong implication that the underlying manifold $X$ is homeomorphic to the sphere or a connected sum of the projective planes. Therefore, we can slightly generalize Theorem 2.7 as follows:

Theorem 2.10. If $X$ is a compact connected oriented manifold with (i) $b_{1}=0$ and $\tau \leqslant 1$, and 
(ii) a self-dual metric with positive scalar curvature, then it is conformally equivalent to the Euclidean 4-sphere or the projective plane with Fubini-Study metric.

\section{The system $\left|-\frac{1}{2} K\right|$, when $\tau=2$}

In this section, we only study the case when $\tau=2$.

From the last section, we see that the obstruction for the associated map being an embedding is essentially from the curves $C$ such that $-\frac{1}{2} K \cdot C=0$. Let us call any such irreducible curve an exceptional curve and let $\mathbb{E}$ be the set of all points on exceptional curves. It is easy to check that exceptional curves are nonsingular rational.

To locate the exceptional curves, we use the bundle $\mathbf{D}_{\varepsilon_{1} \varepsilon_{2}}$. This bundle has nontrivial holomorphic sections. In fact, the system $\left|-\frac{1}{4} K+\frac{1}{2} \varepsilon_{1} \alpha_{1}+\frac{1}{2} \varepsilon_{2} \alpha_{2}\right|$ has dimension at least 1 . Elements in this system will be denoted by $D_{\varepsilon_{1} \varepsilon_{2}}$. To save space we shall call this system $\left(\varepsilon_{1}, \varepsilon_{2}\right)$. Since $-\frac{1}{4} K \cdot L=1, \alpha_{1} \cdot L=0$ and $\alpha_{2} \cdot L=0$, one can easily imitate the techniques in the proof of Lemmas 2.4 and 2.5 to see that every element in the system $\left(\varepsilon_{1}, \varepsilon_{2}\right)$ is a rational surface. In fact, it is the blowing up of a ruled surface once or the projective plane twice. A useful observation is that each $D_{\varepsilon_{1} \varepsilon_{2}}$ contains a unique real twistor line. Let us prove that for $(1,1)$ : If $D_{11}$ does not contain any real twistor line, then every real twistor line interests $D_{11}$ transversely at one point because $D_{11} \cdot L=1$. Therefore $D_{11}$ is diffeomorphic to the parameter space of real twistor lines $X$ via the twistor projection. But the Euler characteristic of $X$ is equal to 4 and the Euler characteristic of $D_{11}$ is equal to 5 . Hence $D_{11}$ must contain some real twistor line $L$. As the self-intersection number of $L$ on the surface $D_{11}$ can be proved to be 1 while distinct real twistor lines are disjoint, then the Hodge index theorem [16] provides the uniqueness.

Now let us fix one $D_{11}$. For any $\left(\varepsilon_{1}, \varepsilon_{2}\right)$, we have the exact sequence:

$$
0 \rightarrow \mathcal{O}\left(\mathbf{D}_{\varepsilon_{1} \varepsilon_{2}} \otimes \mathbf{D}_{11}^{-1}\right) \rightarrow \mathcal{O}\left(\mathbf{D}_{\varepsilon_{1} \varepsilon_{2}}\right) \rightarrow \mathcal{O}_{D_{11}}\left(\mathbf{D}_{\varepsilon_{1} \varepsilon_{2}}\right) \rightarrow 0 \text {. }
$$

When $\left(\varepsilon_{1}, \varepsilon_{2}\right) \neq(1,1)$, we can use Lemma 2.1 to check that $\mathbf{D}_{\varepsilon_{1} \varepsilon_{2}} \otimes \mathbf{D}_{11}^{-1}$ does not have sections. Therefore any nontrivial section of $D_{\varepsilon_{1} \varepsilon_{2}}$ is uniquely restricted to be a nontrivial section of $\mathbf{D}_{\varepsilon_{1} \varepsilon_{2}}$ over $D_{11}$. In case $\left(\varepsilon_{1}, \varepsilon_{2}\right)=(1,1), \mathbf{D}_{11}$ on $D_{11}$ still has nontrivial sections. Let us use $D_{\varepsilon_{1} \varepsilon_{2}}^{\prime}$ to denote a divisor on $D_{11}$ defined by this restriction. As $c_{1} \cdot \alpha_{j}^{2}=-4, c_{2} \cdot \alpha_{j}=0$, and, $c_{1}^{2} \cdot \alpha_{j}=0$, then on the surface $D_{11}$, we have

$$
\begin{aligned}
D_{\varepsilon_{1} \varepsilon_{2}}^{\prime} \cdot D_{\lambda_{1} \lambda_{2}}^{\prime} & =\frac{1}{8}\left(\frac{1}{2} c_{1}+\alpha_{1}+\alpha_{2}\right)\left(\frac{1}{2} c_{1}+\varepsilon_{1} \alpha_{1}+\varepsilon_{2} \alpha_{2}\right)\left(\frac{1}{2} c_{1}+\lambda_{1} \alpha_{1}+\lambda_{2} \alpha_{2}\right) \\
& =\frac{1}{2}-\frac{1}{4} \sum_{j=1}^{2}\left(\varepsilon_{j} \lambda_{j}+\varepsilon_{j}+\lambda_{j}\right)
\end{aligned}
$$




$$
\begin{aligned}
K_{11} \cdot D_{\varepsilon_{1} \varepsilon_{2}}^{\prime} & =\frac{1}{8}\left(\frac{1}{2} c_{1}+\alpha_{1}+\alpha_{2}\right)\left(-\frac{3}{2} c_{1}+\alpha_{1}+\alpha_{2}\right)\left(\frac{1}{2} c_{1}+\varepsilon_{1} \alpha_{1}+\varepsilon_{2} \alpha_{2}\right) \\
& =-2+\frac{1}{2}\left(\varepsilon_{1}+\varepsilon_{2}\right),
\end{aligned}
$$

where $K_{11}$ is the canonical class of the surface $D_{11}$.

Suppose that $C$ is an exceptional curve such that $\alpha_{1} \cdot C=0$ and $\alpha_{2} \cdot C=0$. Let $D_{11}$ be an element in $(1,1)$ such that $D_{11}$ intersects $C$. This is possible because the system $(1,1)$ has nonzero dimension. But $D_{11} \cdot C=0$. Then the curve $C$ is actually contained in the surface $D_{11}$. On $D_{11}$, we have $K_{11} \cdot C=0$. Now by the adjunction formula and the rationality of $C$, we have $C^{2}=-2$. Since $D_{11}$ is the blowing up of a rational ruled surface once or the projective plane twice, the existence of the curve $C$ on $D_{11}$ implies that this surface is the blowing up of the Hirzebruch surface $F_{2}$ once away from the infinity section $E_{\infty}$ and $E_{\infty}$ is exactly the curve $C$. If $E_{0}$ is the divisor class of the zero section of $F_{2}, F$ is that of a fiber of the ruling and $E$ is the exceptional divisor of the blowing-up, then every divisor class on $D_{11}$ is a linear integral combination of $E_{0}, F$ and $E$ (see [16]). For example,

$$
C=E_{\infty}=E_{0}-2 F \quad \text { and } \quad K_{11}=-2 E_{0}+E .
$$

Let $D_{1-1}^{\prime}=a E_{0}+b F+c E$ for some integers $a, b$ and $c$. Then

$$
0=D_{1-1} \cdot C=D_{1-1}^{\prime} \cdot E_{\infty}=\left(a E_{0}+b F+c E\right) \cdot\left(E_{0}-2 F\right)=b .
$$

But this is not compatible with the consequence of (3.1) and (3.2) that $0=\left(D_{1-1}^{\prime}\right)^{2}=2 a^{2}+2 a b-c^{2}$ and $-2=K_{11} \cdot D_{1-1}^{\prime}=-4 a-2 b-c$. Therefore, not both of $\alpha_{1} \cdot C$ and $\alpha_{2} \cdot C$ are equal to zero. Then we can always choose an $\left(\varepsilon_{1}, \varepsilon_{2}\right), \varepsilon_{i}= \pm 1$, such that

$$
\left(-\frac{1}{4} K+\frac{1}{2} \varepsilon_{1} \alpha_{1}+\frac{1}{2} \varepsilon_{2} \alpha_{2}\right) \cdot C<0 .
$$

It implies that the curve is in the base locus of the system $\left(\varepsilon_{1}, \varepsilon_{2}\right)$. Now it is obvious that exceptional curves cannot be real because when $\bar{C}$ is the conjugate of $C$,

$$
\left(-\frac{1}{4} K+\frac{1}{2} \varepsilon_{1} \alpha_{1}+\frac{1}{2} \varepsilon_{2} \alpha_{2}\right) \cdot \bar{C}=\left(\frac{1}{4} K-\frac{1}{2} \varepsilon_{1} \alpha_{1}-\frac{1}{2} \varepsilon_{2} \alpha_{2}\right) \cdot C>0 .
$$

Suppose that $y$ is a point in the base locus of the system $\left(\varepsilon_{1}, \varepsilon_{2}\right)$ but is not on the curve $C$. Then it cannot be on the unique real twistor line $L$ on the surface $D_{\varepsilon_{1} \varepsilon_{2}}$. For otherwise, this curve $L$ would have been in the base locus of the system $\left(\varepsilon_{1}, \varepsilon_{2}\right)$. Let $L^{\prime}$ be the real twistor line through $y$, it is disjoint from the curve $C$. Through any other point $y^{\prime}$ on $L^{\prime}$, there is a $\hat{D}_{\varepsilon_{1} \varepsilon_{2}}$ in $\left(\varepsilon_{1}, \varepsilon_{2}\right)$ containing $y^{\prime}$. Then $\hat{D}_{\varepsilon_{1} \varepsilon_{2}}$ must contain the twistor line $L^{\prime}$ because it intersects $L^{\prime}$ at more than one point. But the unique real twistor line on $\hat{D}_{\varepsilon_{1} \varepsilon_{2}}$ must intersect the curves $C$ and $\bar{C}$. This cannot be $L^{\prime}$. This contradiction shows that $C$ is precisely the base locus of $\left(\varepsilon_{1}, \varepsilon_{2}\right)$. From the number of choices of $\varepsilon_{1}$ and 
$\varepsilon_{2}$, we see that the set $\mathbb{E}$ consists of at most four curves and $\Phi(\mathbb{E})$ is a set of at most four points. Now we are ready to prove the following:

Proposition 3.1. (i) If $z \in Z \backslash \mho$ and $y \in Z$ such that $z \neq y$, then $\Phi(z) \neq$ $\Phi(y)$.

(ii) $\Phi$ on $Z \backslash \mathbb{C}$ is an embedding.

Proof. Let $S$ be a nonsingular irreducible real element in $\left|-\frac{1}{2} K\right|$ containing z. Its existence is guaranteed by Lemma 2.4. This is a degenerate Del Pezzo surface. If $y$ is not on $S$, then $\Phi(z) \neq \Phi(y)$. Suppose that $y$ is also on $S$. According to Demazure [9], because we blow up only a few points on the plane to $S$ and $z$ is not on any irreducible rational curve $C$ with self-intersection $C^{2}=-2-K_{S} \cdot C=-2$, the system $\left|-K_{S}\right|_{z}$ of elements in $\left|-K_{S}\right|$ containing $z$ has no base points. Then there is a section of $\mathbf{K}_{S}^{-1}$ which vanishes at $z$ but not at $y$. As this section can be extended to be a section of $\mathbf{K}^{-1 / 2}$ on the twistor space, $\Phi(z) \neq \Phi(y)$.

To prove that $\Phi$ is an embedding module $\mathbb{E}$, we pick up any point $z$ in $Z \backslash$ 『; then $\Phi(z)$ is not in the set $\Phi($ ( ) $)$. As $\Phi($ ( $)$ is only a set of finitely many points, a generic element in $\left|-\frac{1}{2} K\right|$ passing through $z$ is disjoint from the set c5. Let us pick up a real one, $S$. This is a surface transversely intersecting the real twistor line through $z$ at the points $z$ and $\bar{z}$. As the surface $S$ is disjoint from the set $\mathbb{E}$, any irreducible curve $D$ on $S$ has the property that $-K_{S} \cdot D>0$. Now one can prove that the associated map of the anticanonical system on $S$ is an embedding. Therefore, the map $\Phi$ is an embedding on $S$. Since $\Phi$ is easily seen to be an embedding on every real twistor line and $\Phi$ is proved to be one-to-one module $\sqrt{5}$, the differential of $\Phi$ at the point $z$ has full rank.

Knowing that $\Phi$ is an embedding modulo $\sqrt{ }$ and that $\sqrt{ }$ is a set of at most four curves, we can apply Siu's vanishing theorem [31] on the bundles $\mathbf{K}^{-1 / 2} \cong \Phi^{*} \mathbf{H}$ and $\mathbf{K}^{-1} \cong \Phi^{*} \mathbf{H}^{2}$ to see that, for all $p \geqslant 1$,

$$
h^{p}\left(Z, \mathcal{O}\left(\mathbf{K}^{-1 / 2}\right)\right)=0 \text { and } h^{p}\left(Z, \mathcal{O}\left(\mathbf{K}^{-1}\right)\right)=0 .
$$

Then from the Riemann-Roch formula, we deduce that

$$
h^{0}\left(Z, \mathcal{O}\left(\mathbf{K}^{-1 / 2}\right)\right)=6 \text { and } h^{0}\left(Z, \mathcal{O}\left(\mathbf{K}^{-1}\right)\right)=19 .
$$

Therefore, the associated map sends the twistor space into $\mathbf{C P}^{5}$. But $h^{0}\left(\mathbf{C P}^{5}, \mathcal{O}\left(\mathbf{H}^{2}\right)\right)=21, \Phi(Z)$ has to be contained in the zeros of two sections of $\mathbf{H}^{2}$, i.e. the intersection of two quadrics.

Now we can also tell the precise number of exceptional curves: Suppose that $D_{11}$ does not contain any exceptional curves. Then $D_{11}$ is disjoint from any exceptional curves because if $C$ is an exceptional curve such that $D_{11} \cdot C>0$, then $D_{11} \cdot \bar{C}<0$. Therefore, $\Phi$ is restricted to be an embedding on $D_{11}$. As one can use an exact sequence argument and various vanishing theorems to 
check that $h^{0}\left(D_{11}, \mathcal{O}_{D_{11}}\left(\mathrm{~K}^{-1 / 2}\right)\right)$ is equal to $4, \Phi\left(D_{11}\right)$ is regarded as a divisor in $\mathbf{C P}^{3}$. Let $n$ be the natural number such that $\Phi\left(D_{11}\right)=n H$, where $H$ is the hyperplane class in projective space. Then the canonical class of the surface $\Phi\left(D_{11}\right)$ is $(n-4) H$. Hence $c_{1}^{2}\left(D_{11}\right)=n(n-4)^{2}$. On the other hand, we can show that $c_{1}^{2}\left(D_{11}\right)=7$. This contradiction shows that each $D_{11}$ must contain an exceptional curve. As the system $(1,1)$ has dimension at least 1 and there are only finitely many exceptional curves, we can always find two distinct elements in the system to contain the same exceptional curve $C$. Suppose that $\left(\alpha_{1}+\alpha_{2}\right)$ . $C \geqslant 0$, then $\left(\alpha_{1}+\alpha_{2}\right) \cdot \bar{C} \leqslant 0$. Hence within the pencil generated by the above two elements in the system $(1,1)$, we can find a particular $D_{11}$ to contain both $C$ and $\bar{C}$. It follows that every real twistor line through $C$ and $\bar{C}$ intersects this $D_{11}$ at least twice and hence is contained in $D_{11}$. This is impossible. Therefore, $\left(\alpha_{1}+\alpha_{2}\right) \cdot C<0$. A similar argument shows that, for any $D_{11}$ in $(1,1), D_{11} \cdot C=-1$ and hence $D_{11} \cdot \bar{C}=1$, i.e. $\left(\alpha_{1}+\alpha_{2}\right) \cdot C=-2$. Now it is easy to prove that the system $(1,1)$ is a pencil because through each point of $\bar{C}$, there is a unique element in $(1,1)$. Regarding the curve $C$ as the intersection of any two elements in $(1,1)$, we have $D_{11}^{\prime}=C$ on a particular $D_{11}$. From (3.1), we have

$$
D_{1-1}^{\prime} \cdot C=D_{1-1}^{\prime} \cdot D_{11}^{\prime}=0
$$

i.e. $\left(\alpha_{1}-\alpha_{2}\right) \cdot C=0$. Therefore, $\alpha_{1} \cdot C=1-1, \alpha_{2} \cdot C=-1$.

Working on the system $(1,-1)$, we obtain another exceptional curve $D$ with $\alpha_{1} \cdot D=-1, \alpha_{2} \cdot D=1$. As the twistor space does not contain real points, exceptional curves are disjoint from their conjugate. As $C$ is in the base locus of the system $(1,1)$, if the curves $C$ and $D$ intersect, then the equation $D_{11} \cdot D=0$ implies that $D$ is also in the base locus of $(1,1)$. This is impossible. Similarly, we see that $C$ and $\bar{D}$ are also disjoint. As a conclusion, there are four exceptional curves, namely $C, \bar{C}, D$, and $\bar{D}$. They are mutually disjoint.

Proposition 3.2. The system $(1,1)$ is a pencil such that

(i) if $D_{11}$ is the unique element in $(1,1)$ containing the curve $D$ (resp. $\left.\bar{D}\right)$, and $L$ is the unique real twistor line on $D_{11}$, then $\Phi\left(D_{11}\right)$ is a 2-dimensional cone over the conic $\Phi(L)$ with $\Phi(D)$ (resp. $\Phi(\bar{D})$ ) as vertex;

(ii) a generic element is the blowing up of the projective plane at two distinct point. It is sent by the associated map to a smooth quadric surface.

Proof. Without loss of generality, we may assume that $D_{11}$ is the blowing up of the Hirzebruch surface $F_{k}$ once away from the infinity section $E_{\infty}$. Let $E_{0}$ be the proper transform of a generic zero section, $F$ a generic fiber and $E$ the exceptional divisor of blowing up, then $F-E=\hat{F}$ is the proper transform of the fiber through the point of blowing-up and the canonical class $K_{11}$ is 
given by

$$
K_{11}=-2 E_{0}+(k-2) F+E .
$$

When $L$ is the unique real twistor line on $D_{11}$, then $K_{11} \cdot L=-3$ and $L^{2}=1$. As $L$ is a nonsingular irreducible curve different from the exceptional divisors $\hat{F}, E$, and the $E_{\infty}$, there are nonnegative integers $a, b$, and $c$ such that

$$
L=a E_{0}+b F-c F .
$$

From the above equalities, we have

$$
\begin{gathered}
a^{2} k+2 a b-c^{2}=1, \\
-a k-2 a-2 b+c=-3 .
\end{gathered}
$$

Eliminating $b$ from these equations, we have

$$
2 a^{2}-(3+c) a+c^{2}+1=0 .
$$

On the other hand, $C$ is an exceptional divisor on $D_{11}$. As the curve $C$ intersects the real twistor line at one point, it is easy to use (3.3) and (3.4) to deduce that when $k=1, C$ cannot be $E_{\infty}$. Therefore, in any case, $C$ is either $E$ or $\hat{F}$.

To determine $L$ and $C$ in terms of $E_{0}, F$, and $E$, we first claim that $D_{-1-1}^{\prime}=L$. This can easily be seen once we regard $D_{-1-1}^{\prime}$ as the intersection of the surface $D_{11}$ and its conjugate $D_{-1-1}$. Considering the curve $C$ as the intersection of two elements in the system $(1,1)$, we see that $D_{11}^{\prime}=C$. Therefore,

$$
-\frac{1}{2} K_{\mid D_{11}}+D_{-1-1}^{\prime}=C+L .
$$

Suppose that $C=E$. From (3.1), we have

$$
1=D_{11}^{\prime} \cdot D_{-1-1}^{\prime}=C \cdot L=E \cdot\left(a E_{0}+b F-c E\right)=c .
$$

This is substituted into (3.5) to show that $a=1$. Then from (3.4), we deduce that $2 b=2-k$. As $b \geqslant 0$ and $k \geqslant 1$, then $b=0$ and $k=2$, i.e. $L=E_{0}-E$ and the surface $D_{11}$ is the blowing up of $F_{2}$ once away from the infinity section. Moreover, $-\frac{1}{2} K_{\mid D_{11}}=C+L=E_{0}$. Hence $-\frac{1}{2} K \cdot E_{\infty}=0$. Therefore, $E_{\infty}$ is an exceptional curve in the twistor space. As it is different from $C$ and $\bar{C}$, it is either $D$ or $\bar{D}$.

Conversely, if $D_{11}$ is the unique element in the pencil containing $D$, then by rationality of $D$ and the adjunction formula on $D_{11}$, we have

$$
D^{2}=-2-K_{11} \cdot D=-2 \text {. }
$$


Therefore, $D_{11}$ is the blowing up of $F_{2}$ once away from the infinity section which is the curve $D$. As $C$ is an exceptional divisor on $D_{11}$ and disjoint from the infinity section $D$, then $C=E$. Now, it is easy to see that this kind of $\Phi\left(D_{11}\right)$ is a simple cone described by assertion (i).

For generic $D_{11}$, we must have $C=\hat{F}=F-E$. One may carry out the similar elementary computation to prove assertion (i), we do not repeat the calculation here.

Remark 3.3. Of course, for $\left(\varepsilon_{1}, \varepsilon_{2}\right)=(1,-1),(-1,1)$ or $(-1,-1)$, the system $\left(\varepsilon_{1}, \varepsilon_{2}\right)$ can also be described by this proposition with $D$ and $\bar{D}$ appropriately changed. One particular consequence of this proposition is that the images of distinct exceptional curves are distinct points. Then from Proposition 3.1, we see that there are exactly two conjugate pairs of singular points on $\Phi(Z)$. Through each singular point of $\Phi(Z)$, there is a pair of simple cones with this point as vertex and a pair of simple cones with this point as a nonsingular point. We shall use this remark to give a complete algebraic description of $\Phi(Z)$.

Theorem 3.4. If $X$ is a compact connected simply connected oriented self-dual Riemannian manifold with positive scalar curvature and signature 2 , then $X$ is diffeomorphic to $\mathbf{C P}^{2} \# \mathbf{C} \mathbf{P}^{2}$.

Proof. Let $D_{11}$ be a generic element in $(1,1)$. It is the blowing up of $\mathbf{C P}^{2}$ at two distinct points. The unique real twistor line $L$ on $D_{11}$ is the proper transform of a line away from the two points. As any other real twistor lines intersects $D_{11}$ transversely at one point, the twistor fibration is restricted to be a smooth map from $D_{11}$ onto $X$ such that $\pi: D_{11} / L \rightarrow X /\{x\}, x=\pi(L)$, is a diffeomorphism. This projection is orientation reversing because the horizontal orientation on $Z$ is opposite to the one on $X$. Now as $L$ is a rational curve on the surface $D_{11}$ with self-intersection 1 and $D_{11}$ is diffeomorphic to $\mathbf{C P}^{2} \# \overline{\mathbf{C P}}^{2} \# \overline{\mathbf{C P}}^{2}$, it is clear that $X$ is diffeomorphic to $\mathbf{C P} \mathbf{P}^{2} \# \mathbf{C P}^{2}$.

To study the image of the twistor space in $\mathbf{C P}^{5}$, let $Q_{0}$ and $Q_{\infty}$ be two quadrics in $\mathrm{CP}^{5}$ such that their intersection contains $\Phi(Z)$. Since a generic real element in $\left|-\frac{1}{2} K\right|$ is disjoint from the four exceptional curves, it is a nonsingular complete intersection of two smooth quadrics in $\mathbf{C P}^{4}$. Therefore, a generic element in the pencil generated by $Q_{0}$ and $Q_{\infty}$ has rank at least 5 . As $\Phi(Z)$ is an irreducible variety in $Q_{0}$ and $Q_{0}$ can be assumed to have rank at least 5, a theorem of Hodge and Pedoe (p. 244 in [23]) shows that $\Phi(Z)=Q_{0}$ $\cap Q_{\infty}$. In particular, $Q_{0} \cap Q_{\infty}$ has exactly four singular points and $Z$ can be regarded as a small resolution of the singularity of $Q_{0}$ and $Q_{\infty}$. From Proposition 3.2, we know that through each singular point on $Q_{0}$ and $Q_{\infty}$, there are nonsingular quadric surfaces contained in $Q_{0}$ and $Q_{\infty}$. This observation enables one to argue that the vertex of a rank 5 element in the pencil 
generated by $Q_{0}$ and $Q_{\infty}$ is not contained in the intersection. Therefore, one may follow Hodge and Pedoe's elementary transformation on coordinates ( $p$. 281 in [23]) to see that a generic member in the pencil is smooth. Then we assume that $Q_{0}$ and $Q_{\infty}$ are smooth.

There are only two types of intersection of two full rank quadrics in $\mathbf{C P}^{5}$ with four singular points. One can find a homogeneous coordinate $\left[w_{0}: \cdots: w_{5}\right]$ on $\mathbf{C P}^{5}$ such that they are either

or

$$
\begin{aligned}
& Q_{0}=\left\{w \in \mathbf{C P}^{5}: a w_{0}^{2}+a w_{1}^{2}+b w_{2}^{2}+b w_{3}^{2}+c w_{4}^{2}+d w_{5}^{2}=0\right\}, \\
& Q_{\infty}=\left\{w \in \mathbf{C P}^{5}: w_{0}^{2}+w_{1}^{2}+w_{2}^{2}+w_{3}^{2}+w_{4}^{2}+w_{5}^{2}=0\right\} \\
& Q_{0}=\left\{w \in \mathbf{C P}^{5}: 2 a w_{0} w_{1}+w_{1}^{2}+2 b w_{2} w_{3}+w_{3}^{2}+c w_{4}^{2}+c w_{5}^{2}=0\right\}, \\
& Q_{\infty}=\left\{w \in \mathbf{C} \mathbf{P}^{5}: 2 w_{0} w_{1}+2 w_{2} w_{3}+w_{4}^{2}+w_{5}^{2}=0\right\},
\end{aligned}
$$

where $a, b, c, d$ are distinct nonzero (complex) numbers. In the pencil generated by the second pair of quadrics, there is only rank 4 quadric. The line of vertices of this quadric interests the other members of the pencil at two points. These points are singular points of the intersection. One may use projective geometry argument to check that there is no 2-dimensional simple cone contained in the intersection and passing through any one of these two points. In view of Remark 3.3, we can conclude that $\Phi(Z)$ is the intersection of the first pair of quadrics.

In the pencil generated by the first pair of quadrics, there are two rank 4 quadrics and two rank 5 quadrics. To study the real structure on this pencil, we must first recall that $Z$ is a real space without real point. Secondly, the target space $\mathbf{C P}^{5}$ of the associated map is the projectivization of the dual of the real vector space $H^{0}\left(Z, \mathcal{O}\left(\mathbf{K}^{-1 / 2}\right)\right)$, therefore $\mathbf{C P}$ has an induced real structure. Then through the map $\Phi$ which is an embedding modulo $\mathbb{E}, \Phi(Z)$ becomes a real space without real point. The four singular points on $\Phi(Z)$ are two conjugate pairs. They are lying on the lines of vertices of the two rank 4 quadrics. In fact, the two rank 4 quadrics are real and hence their lines of vertices are joining a conjugate pair of singular points, otherwise, according to Remark 3.3, the lines of vertices would have been in the intersection. The two rank 5 quadrics are also real, otherwise the intersection would have contained real points. From the reality of these singular elements, one can easily change from the $w$-coordinate to a homogeneous coordinate $\left[y_{0}: \cdots: y_{5}\right]$ such that the real structure is simply the usual conjugation, and then one shifts the parameter of the pencil so that $\Phi(Z)=Q_{0} \cap Q_{\infty}$ with

$$
\begin{aligned}
& Q_{0}=\left\{y \in \mathbf{C P}^{5}: \lambda_{1}\left(y_{0}^{2}+y_{1}^{2}\right)+\lambda_{2} y_{2}^{2}-\lambda_{3} y_{3}^{2}-\lambda_{4}\left(y_{4}^{2}+y_{5}^{2}\right)=0\right\}, \\
& Q_{\infty}=\left\{y \in \mathbf{C P}^{5}: y_{0}^{2}+y_{1}^{2}+y_{2}^{2}-y_{3}^{2}-y_{4}^{2}-y_{5}^{2}=0\right\},
\end{aligned}
$$


where the $\lambda_{j}$ 's are distinct real numbers. Certainly, we can choose $\lambda_{4}=1$. When $\lambda_{1}$ is assumed to be bigger than $\lambda_{4}$, the requirement that $Q_{0} \cap Q_{\infty}$ not contain real points will force us to have

$$
\lambda_{1}>\lambda_{2}>\lambda_{3}>\lambda_{4}=1 \text {. }
$$

Then a real mobius transform on the parameter of the pencil can take $\lambda_{1}$ to 2 and $\lambda_{3}$ to $\frac{3}{2}$. We are allowed only one degree of freedom-the $\lambda_{2}$ with $2>\lambda_{2}>\frac{3}{2}$. For future reference, we change coordinate again and have

Theorem 3.5. If $X$ is a compact connected simply connected oriented self-dual Riemannian manifold with positive scalar curvature and signature 2 , then its twistor space $Z$ is a resolution of the intersection of two quadrics:

$$
\begin{aligned}
& Q_{0}=\left\{z \in \mathbf{C P}^{5}: 2\left(z_{0}^{2}+z_{1}^{2}\right)+\lambda z_{2}^{2}+\frac{3}{2} z_{3}^{2}+z_{4}^{2}+z_{5}^{2}=0\right\}, \\
& Q_{\infty}=\left\{z \in \mathbf{C P}^{5}: z_{0}^{5}+z_{1}^{2}+z_{2}^{2}+z_{3}^{2}+z_{4}^{2}+z_{5}^{2}=0\right\}
\end{aligned}
$$

with $2>\lambda>\frac{3}{2}$.

The resolution $\Phi: Z \rightarrow Q_{0} \cap Q_{\infty}$ is a holomorphic embedding modulo four curves. Under this map, the real structure $Z$ is an extension of the real structure $J: \mathbf{C}^{6} \rightarrow \mathbf{C}^{6}$ defined by

$$
J\left(z_{0}, z_{1}, \cdots, z_{5}\right)=\left(\bar{z}_{0}, \bar{z}_{1}, \bar{z}_{2},-\bar{z}_{3},-\bar{z}_{4},-\bar{z}_{5}\right) .
$$

\section{Self-duality on $\mathrm{CP}^{2} \# \mathrm{CP}^{2}$}

In this section and the next, we change our viewpoint. Our aim is to construct a self-dual conformal class on $\mathbf{C P}^{2} \# \mathbf{C} \mathbf{P}^{2}$ associated to every real number $\lambda$ in the open interval $\left(\frac{3}{2}, 2\right)$, and then show that each such conformal class contains a metric with positive scalar curvature. To set up the construction, we follow Hurtubise [24].

Let $\lambda$ be a real number such that $2>\lambda>\frac{3}{2}$. We put $\lambda_{1}=2, \lambda_{2}=\lambda, \lambda_{3}=\frac{3}{2}$, $\lambda_{4}=1$ and fix a homogeneous coordinate $z=\left[z_{0}: \cdots: z_{5}\right]$ on CP. Then we define a pair of quadrics $Q_{0}$ and $Q_{\infty}$ by (3.6) and a real structure on $\mathbf{C P}^{5}$ by (3.7). From the last section, we know that nonsingular real conics are candidates of real twistor lines. As every nonsingular conic is contained in a unique plane and a plane is contained in a member of the pencil, we want to parametrize this kind of plane: For any $t$ in $\mathbf{C}$, let $\gamma_{j}$ be the function $\gamma_{j}(t)=\left(\lambda_{j}+t\right)^{1 / 2}$; for any $\sigma=\left[\sigma_{0}: \sigma: \sigma_{2}: \sigma_{3}\right]$ in $\mathbf{C P}^{3}$, we define the matrices 
$A(\sigma ; t)$ and $B(\sigma ; t)$ by

$$
\begin{aligned}
& \left|\begin{array}{rrrrrr}
\gamma_{1} \sigma_{1} & -i \gamma_{1} \sigma_{1} & \gamma_{2} \sigma_{2} & -i \gamma_{3} \sigma_{2} & \gamma_{4} \sigma_{3} & -i \gamma_{4} \sigma_{3} \\
\gamma_{1} \sigma_{0} & -i \gamma_{1} \sigma_{0} & \gamma_{2} \sigma_{3} & i \gamma_{3} \sigma_{3} & -\gamma_{4} \sigma_{2} & -i \gamma_{4} \sigma_{2} \\
-\gamma_{1} \sigma_{3} & -i \gamma_{1} \sigma_{3} & \gamma_{2} \sigma_{0} & -i \gamma_{3} \sigma_{0} & \gamma_{4} \sigma_{1} & i \gamma_{4} \sigma_{1} \\
\gamma_{1} \sigma_{2} & i \gamma_{1} \sigma_{2} & -\gamma_{2} \sigma_{1} & -i \gamma_{3} \sigma_{1} & \gamma_{4} \sigma_{0} & -i \gamma_{4} \sigma_{0}
\end{array}\right| \text {, } \\
& \left|\begin{array}{rrrrrr}
\gamma_{1} \sigma_{1} & i \gamma_{1} \sigma_{1} & \gamma_{2} \sigma_{2} & i \gamma_{3} \sigma_{2} & \gamma_{4} \sigma_{3} & i \gamma_{4} \sigma_{3} \\
\gamma_{1} \sigma_{0} & i \gamma_{1} \sigma_{0} & \gamma_{2} \sigma_{3} & -i \gamma_{3} \sigma_{3} & -\gamma_{4} \sigma_{2} & i \gamma_{4} \sigma_{2} \\
-\gamma_{1} \sigma_{3} & i \gamma_{1} \sigma_{3} & \gamma_{2} \sigma_{0} & i \gamma_{3} \sigma_{0} & \gamma_{4} \sigma_{1} & -i \gamma_{4} \sigma_{1} \\
\gamma_{1} \sigma_{2} & -i \gamma_{1} \sigma_{2} & -\gamma_{2} \sigma_{1} & i \gamma_{3} \sigma_{1} & \gamma_{4} \sigma_{0} & i \gamma_{4} \sigma_{0}
\end{array}\right| \text {. }
\end{aligned}
$$

For $t=\infty$, we put $\gamma_{j}(\infty) \equiv 1$ for each $j$. These so-called $A$-matrices and $B$-matrices are considered as linear transformations from $\mathbf{C}^{6}$ to $\mathbf{C}^{4}$. The projectivization of their kernels are in $Q_{t}$, where $Q_{t}$ is given by $Q_{0}+t Q_{\infty}$. These projective subspaces in $\mathbf{C P}^{5}$ are also denoted by $A(\sigma ; t)$ and $B(\sigma ; t)$. Except when $t=-\lambda_{1}$ and $t=-\lambda_{4}$, the families of $A(\sigma ; t)$ and of $B(\sigma ; t)$ give all planes in $Q_{t}$. When $t=-\lambda_{1}$ or $-\lambda_{4}$, the two families of projective subspaces coincide but some members in the family are 3-dimensional, some are 2dimensional. Though not every plane in $Q_{-\lambda_{1}}$ or $Q_{-\lambda_{4}}$ is in these families, every plane in $Q_{t}$ containing the line of vertices of $Q_{t}$ is in this family.

For any $\sigma$ and $t$, whenever $A(\sigma ; t)$ is a plane, we call it an $\alpha$-plane. This plane intersects $Q_{0} \cap Q_{\infty}$ along a conic. This conic is called an $\alpha$-conic and is denoted by $\alpha(\sigma ; t)$. Similarly, one uses $B(\sigma ; t)$ to define $\beta$-planes and $\beta$-conics. As a nonsingular conic in $Q_{0} \cap Q_{\infty}$ is real if and only if its plane is real, one can easily check that an $\alpha$-conic $\alpha(\sigma ; t)$ (resp. $\beta(\sigma ; t))$ is real if and only if

(i) $t$ is a real number in an interval $I$ in $\mathbf{R} \cup\{\infty\} \cong \mathbf{R P}^{\mathbf{1}}$ :

$$
I=\left(-\infty,-\lambda_{1}\right] \cup\left[-\lambda_{4}, \infty\right) \cup\{\infty\}
$$

and

(ii) $\sigma$ is in a copy of $\mathbf{R P}^{3}$ in $\mathbf{C P}^{3}$ given by

$$
\left[\sigma_{0}: \sigma_{1}: \sigma_{2}: \sigma_{3}\right]=\left[\bar{\sigma}_{2}:-\bar{\sigma}_{3}: \bar{\sigma}_{0}:-\bar{\sigma}_{1}\right] \text {. }
$$

Since every real plane containing a singular point of $Q_{0} \cap Q_{\infty}$ is contained in a rank 4 quadric $Q_{-\lambda_{1}}$ or $Q_{-\lambda_{4}}$, and through every nonsingular point on $Q_{0} \cap Q_{\infty}$ there is a unique real $\alpha$-conic (and a unique real $\beta$-conic), the parameter space $A^{0}$ of all real $\alpha$-conics in $Q_{0} \cap Q_{\infty}$ missing the singular points is identified to $\mathbf{R P}^{3} \times I^{0}$, where

$$
I^{0}=\left(-\infty,-\lambda_{1}\right) \cup\left(-\lambda_{4}, \infty\right) \cup\{\infty\} .
$$

Moreover, when $Z^{0}$ is the set of points in $Q_{0} \cap Q_{\infty}$ lying on the $\alpha$-conics parametrized by $A^{0}$, then $Z^{0}$ is an open submanifold in $Q_{0} \cap Q_{\infty}$. It is a real 
space without real points. There is a fibration $\pi: Z^{0} \rightarrow A^{0}$ commuting with the real structure. The fibers are real $\alpha$-conics with normal bundle $\mathbf{H} \oplus \mathbf{H}$ [21]. Given these data, we recall from $\$ 1$ that a self-dual conformal class on $A^{0}$ can be explicitly constructed by taking the real part of the infinitesimal analogue of a family of $\alpha$-conics intersecting at a point.

Note that when $A(\sigma ; t)$ and $A\left(\tau ; t^{\prime}\right)$ are $\alpha$-planes in distinct elements of the pencil, they intersect if and only if their corresponding $\alpha$-conics intersect. Therefore, the algebraic condition that $\alpha(\sigma ; t)$ and $\alpha\left(\tau ; t^{\prime}\right)$ intersect can easily be computed. When we use $\gamma_{j}$ to denote $\left(\lambda_{j}+t\right)^{1 / 2}$ and $\gamma_{j}^{\prime}$ to denote $\left(\lambda_{j}+t^{\prime}\right)^{1 / 2}$, then $\alpha(\sigma ; t)$ and $\alpha\left(\tau ; t^{\prime}\right)$ intersect if and only if $R\left(\sigma, t, \tau, t^{\prime}\right)=0$, where

$$
\begin{aligned}
& R\left(\sigma_{0}, \sigma_{1}, \sigma_{2}, \sigma_{3}, t, \tau_{0}, \tau_{1}, \tau_{2}, \tau_{3}, t^{\prime}\right) \\
& \equiv \gamma_{1} \gamma_{1}^{\prime} \gamma_{4} \gamma_{4}^{\prime}\left(\gamma_{2} \gamma_{3}^{\prime}-\gamma_{3} \gamma_{2}^{\prime}\right)\left(\sigma_{0}^{2} \tau_{2}^{2}+\sigma_{1}^{2} \tau_{3}^{2}+\sigma_{2}^{2} \tau_{0}^{2}+\sigma_{3}^{2} \tau_{1}^{2}\right) \\
&+\left(\gamma_{1}^{2} \gamma_{4}^{\prime}+\gamma_{1}^{\prime} \gamma_{4}^{2}\right)\left(\gamma_{3} \gamma_{2}^{\prime}-\gamma_{2} \gamma_{3}^{\prime}\right)\left(\sigma_{0} \sigma_{2} \tau_{0} \tau_{2}+\sigma_{1} \sigma_{3} \tau_{1} \tau_{3}\right) \\
&+2 \gamma_{1} \gamma_{1}^{\prime}\left(\gamma_{4}^{2} \gamma_{2}^{\prime} \gamma_{3}^{\prime}-\gamma_{4}^{\prime 2} \gamma_{2} \gamma_{3}\right)\left(\sigma_{2} \sigma_{3} \tau_{0} \tau_{1}+\sigma_{0} \sigma_{1} \tau_{2} \tau_{3}\right) \\
&+2 \gamma_{4} \gamma_{4}^{\prime}\left(\gamma_{1}^{\prime 2} \gamma_{2} \gamma_{3}-\gamma_{1}^{2} \gamma_{2}^{\prime} \gamma_{3}^{\prime}\right)\left(\sigma_{1} \sigma_{2} \tau_{0} \tau_{3}+\sigma_{0} \sigma_{3} \tau_{1} \tau_{2}\right) \\
&+\left(\gamma_{1}^{2} \gamma_{4}^{\prime 2}-\gamma_{1}^{\prime 2} \gamma_{4}^{2}\right)\left(\gamma_{2} \gamma_{3}^{\prime}+\gamma_{3} \gamma_{2}^{\prime}\right)\left(\sigma_{1} \sigma_{3} \tau_{0} \tau_{2}+\sigma_{0} \sigma_{2} \tau_{1} \tau_{3}\right) .
\end{aligned}
$$

Let $U_{j}=\left\{\boldsymbol{\sigma} \in \mathbf{C P}^{3}: \sigma_{j} \neq 0\right\}$ and $V_{j}=\left\{\left[a_{0}: a_{1}\right] \in \mathbf{C P}^{1}: a_{j} \neq 0\right\}$. Then $A^{0}$ is covered by the real part of $\left(U_{0} \cup U_{3}\right) \times\left(V_{0} \cup V_{1}\right)$. On $U_{0} \times V_{0}$, let $\zeta_{k}=\sigma_{k} / \sigma_{0}$ for $k=1,2,3 ; t=a_{1} / a_{0}$. In the following computation, we assume that $t \neq-\lambda_{j}$ for $j=1,2,3,4$. To obtain the infinitesimal condition that the $\alpha$-plane

$$
A\left(\zeta_{j} ; t\right) \equiv A\left(\left[1: \zeta_{1}: \zeta_{2}: \zeta_{3}\right] ; t\right)
$$

intersects $A\left(\zeta_{j}+d \zeta_{j} ; t+d t\right)$, we simply make the following substitutions in the equation $R\left(\sigma, t, \tau, t^{\prime}\right)=0$ :

$$
\begin{aligned}
\sigma_{0} & =\tau_{0}=1, \\
\sigma_{j} & =\zeta_{j}, \tau_{j}=\zeta_{j}+d \zeta_{j} \quad \text { for } j=1,2,3, \\
\gamma_{k}^{\prime} & =\gamma_{k}+\frac{d t}{2 \gamma_{k}} \quad \text { for } k=1,2,3,4 .
\end{aligned}
$$

The algebraic condition that $A\left(\zeta_{j} ; t\right)$ and $A\left(\zeta_{j}+d \zeta_{j} ; t+d t\right)$ intersect will involve the derivatives $\left(d \zeta_{j} ; d t\right)$ up to order 3 . But up to order 3 ,

$$
R\left(\zeta_{j}, t, \zeta_{j}+d \zeta_{j}, t+d t\right)=-\frac{d t}{2 \gamma_{2} \gamma_{3}} \Omega\left(\zeta_{j} ; t\right)
$$


where the quadratic differential $\Omega$ at $\left(\zeta_{j} ; t\right)$ is given by

$$
\begin{aligned}
\Omega= & -q\left[\left(d \zeta_{2}\right)^{2}+\zeta_{1}^{2}\left(d \zeta_{3}\right)^{2}+\zeta_{3}^{2}\left(d \zeta_{1}\right)^{2}\right] \\
& -4 p \zeta_{2} d \zeta_{1} d \zeta_{3}+2 q \zeta_{1} \zeta_{3} d \zeta_{1} d \zeta_{3}+2 k \zeta_{3} d \zeta_{1} d \zeta_{2}-2 l \zeta_{1} d \zeta_{2} d \zeta_{3} \\
& +\frac{1}{4}\left(\lambda_{4}-\lambda_{1}\right) f(\zeta) \frac{(d t)^{2}}{\left(\lambda_{1}+t\right)\left(\lambda_{4}+t\right)}
\end{aligned}
$$

$p, q, k, l$ are quadratic polynomials in $t$ :

$$
\begin{aligned}
& p(t)=\left(\lambda_{2}+t\right)\left(\lambda_{3}+t\right)\left(\lambda_{1}-\lambda_{4}\right), \\
& q(t)=\left(\lambda_{1}+t\right)\left(\lambda_{4}+t\right)\left(\lambda_{2}-\lambda_{3}\right), \\
& k(t)=\left(\lambda_{4}+t\right)\left[\lambda_{1}\left(\lambda_{2}+\lambda_{3}\right)-2 \lambda_{2} \lambda_{3}+\left(2 \lambda_{1}-\lambda_{2}-\lambda_{3}\right) t\right], \\
& l(t)=\left(\lambda_{1}+t\right)\left[\lambda_{4}\left(\lambda_{2}+\lambda_{3}\right)-2 \lambda_{2} \lambda_{3}+\left(2 \lambda_{4}-\lambda_{2}-\lambda_{3}\right) t\right],
\end{aligned}
$$

and

$$
\begin{aligned}
f(\zeta)= & \left(\lambda_{4}-\lambda_{1}\right)\left(\lambda_{2}-\lambda_{3}\right)\left(\zeta_{2}^{2}+\zeta_{1}^{2} \zeta_{3}^{2}\right) \\
& +2\left[\left(\lambda_{1}+\lambda_{4}\right)\left(\lambda_{2}+\lambda_{3}\right)-2 \lambda_{1} \lambda_{4}-2 \lambda_{2} \lambda_{3}\right] \zeta_{1} \zeta_{2} \zeta_{3} .
\end{aligned}
$$

Here we see that when $d t$ is nonzero, $A\left(\zeta_{j} ; t\right)$ and $A\left(\zeta_{j}+d \zeta_{j}, t+d t\right)$ intersect if and only if $\Omega_{\left(\zeta_{j} ; t\right)}=0$. This is exactly when their corresponding $\alpha$-planes intersect. The factor $d t$ turns up in (4.2) because two $\alpha$-planes in one quadric always intersect. But it is easy to find the algebraic condition that two $\alpha$-planes in a generic quadric intersect at a point in $Q_{0} \cap Q_{\infty}$. This condition is equivalent to the condition that their corresponding $\alpha$-conics intersect. Then one can check that $\alpha\left(\zeta_{j} ; t\right)$ and $\alpha\left(\zeta_{j}+d \zeta_{j} ; t\right)$ intersect if and only if $\Omega_{\left(\alpha_{j} ; t\right)}=0$ and $d t=0$. Therefore, the quadratic differential $\Omega$ is restricted to the real part of $U_{0} \times V_{0}$ to define a self-dual conformal structure.

From (4.1), we check that $\alpha\left(\zeta_{1}, \zeta_{2}, \zeta_{3}, t\right)$ is a real conic if and only if $t \in I^{0}$, $\left|\zeta_{2}\right|^{2}=1$ and $\zeta_{3}=-\zeta_{2} \bar{\zeta}_{1}$. Then we substitute

$$
\zeta_{1}=x+i y, \quad \zeta_{2}=e^{i \omega}, \quad \zeta_{3}=-e^{i \omega}(x-i y),
$$

where $(x, y) \in R^{2}$ and $\omega \in[0,2 \pi)$, into (4.3). Then in real coordinates

$$
\Omega_{\left(\zeta_{1}, \zeta_{2}, \zeta_{3}, t\right)}=\Omega_{(x, y, \omega, t)}=e^{2 i \omega} h,
$$

where

$$
\begin{aligned}
h= & 4 p\left[(d x)^{2}+(d y)^{2}\right]+4 q(y d x-x d y)^{2} \\
& +4\left[q\left(x^{2}+y^{2}\right)-l\right](y d x-x d y)(d \omega) \\
& +\left\{q\left[1+\left(x^{2}+y^{2}\right)^{2}\right]-2 l\left(x^{2}+y^{2}\right)\right\}(d \omega)^{2} \\
& +\frac{1}{4}\left(\lambda_{1}-\lambda_{4}\right) F \frac{(d t)^{2}}{\left(\lambda_{1}+t\right)\left(\lambda_{4}+t\right)}
\end{aligned}
$$


with

$$
\begin{aligned}
F \equiv & F(x, y)=\left[1+\left(x^{2}+y^{2}\right)^{2}\right]\left(\lambda_{1}-\lambda_{4}\right)\left(\lambda_{2}-\lambda_{3}\right) \\
& +2\left(x^{2}+y^{2}\right)\left[\left(\lambda_{1}+\lambda_{4}\right)\left(\lambda_{2}+\lambda_{3}\right)-2 \lambda_{1} \lambda_{4}-2 \lambda_{2} \lambda_{3}\right] .
\end{aligned}
$$

Note that $F(x, y)$ is a strictly positive function. In fact, if $F(x, y)=0$, the real $\alpha$-conic with parameter $(x, y, \omega, t)$ would have been singular (cf. [24]). But singular real $\alpha$-conics must contain real points in the intersection $Q_{0} \cap Q_{\infty}$. By our choice of the $\lambda$ 's, this is impossible.

One can check that if $t \in\left(-\infty,-\lambda_{1}\right) \cup\left(-\lambda_{4}, \infty\right)$, then $h$ is a positive definite differential. On the real part of $U_{0} \times V_{0}$, denoted by $\left(U_{0} \times V_{0}\right)_{R}$, we define

$$
g=\frac{1}{\left(1+t^{2}\right)} \cdot \frac{1}{\left(1+x^{2}+y^{2}\right)^{2}} \cdot h .
$$

Then $g$ is a self-dual metric on $\left(U_{0} \times V_{0}\right)_{R} \cap A^{0}$ with respect to a certain orientation. One can also work on affine coordinates of $\left(U_{0} \times V_{1}\right)_{R}$, $\left(U_{3} \times V_{0}\right)_{R}$, and $\left(U_{3} \times V_{1}\right)_{R}$ to find the local expression of the self-dual conformal structure and then check that the metric $g$ on $\left(U_{0} \times V_{0}\right)_{R} \cap A^{0}$ is extended to be a metric on $A^{0}$ such that the conformal structure defined by $g$ is $\Omega$. We do not repeat this computation but give the functions of coordinate change: when the affine coordinate on $V_{1}$ is denoted by $\hat{t}$, to change from $V_{0}$ to $V_{1}$ on $V_{0} \cap V_{1}$, one takes $\hat{t}=1 / t$. When the real coordinate on $\left(U_{3}\right)_{R}$ is denoted by $(u, v, \theta)$ with $(u, v) \in R^{2}$ and $\theta \in[0,2 \pi)$, to change from the $(x, y, \omega)$-coordinate to the $(u, v, \theta)$-coordinate, one takes

$$
\begin{aligned}
& u=-\left(x^{2}+y^{2}\right)^{-1}(x \cos \omega+y \sin \omega), \\
& v=\left(x^{2}+y^{2}\right)^{-1}(x \sin \omega-y \cos \omega), \\
& e^{i \theta}=-e^{-i \omega}(x+i y)(x-i y)^{-1} .
\end{aligned}
$$

Apparently, the metric $g$ on $A^{0}$ is not defined when $t$ is equal to $-\lambda_{1}$ or $-\lambda_{4}$, but this is also where $A^{0}$ is not defined. We shall take a coordinate change on $A^{0}$ to extend the metric $g$ onto $\mathbf{C P}^{2} \# \mathbf{C P}^{2}$.

From (3.6), we see that the four singular points on $Q_{0} \cap Q_{\infty}$ are

$$
\begin{array}{ll}
x_{0}=[1: i: 0: 0: 0: 0], & J\left(x_{0}\right)=[1:-i: 0: 0: 0: 0], \\
y_{0}=[0: 0: 0: 0: 1: i], & J\left(y_{0}\right)=[0: 0: 0: 0: 0: 1:-i] .
\end{array}
$$

To resolve the coordinate singularity at $t=-\lambda_{4}$, we first check that the family of real $\alpha$-conics through $x_{0}$ and $J\left(x_{0}\right)$ is parametrized by a 2 -sphere. Within this family, two elements are exceptional in the sense that the 2-dimensional 
simple cones joining these conics to the point $y_{0}$ are in the intersection $Q_{0} \cap Q_{\infty}$. We pick up one of these conics $L$, namely $\alpha\left(\sigma ;-\lambda_{1}\right)$ with $\sigma=$ $[1: 0: 1: 0]$. Let $\Lambda$ be the simple cone over $L$ with $y_{0}$ as vertex. With $y_{0}$ and $L$ removed from $\Lambda$, the resultant space is diffeomorphic to $A^{0}=R P^{3} \times I^{0}$. In our context, this is true because through/every point on $\Lambda \backslash\left(\left\{y_{0}\right\} \cup L\right)$, there is a unique real $\alpha$-conic missing the singular points of $Q_{0} \cap Q_{\infty}$. Now we change the $z$-coordinate on $\mathbf{C P}^{5}$ to an $\eta$-coordinate by defining $\eta=$ $\left[\eta_{1}: \eta_{1}^{\prime}: \eta_{2}: \eta_{2}^{\prime}: \eta_{3}: \eta_{3}^{\prime}\right]$ :

$$
\begin{array}{rlrl}
\eta_{1}= & z_{0}+i z_{1}, & \eta_{1}^{\prime}=z_{0}-i z_{1}, \\
\eta_{2}=\left(\lambda_{1}-\lambda_{2}\right)^{1 / 2} z_{2} & \eta_{2}^{\prime}=\left(\lambda_{1}-\lambda_{2}\right)^{1 / 2} z_{2} \\
& +i\left(\lambda_{1}-\lambda_{3}\right)^{1 / 2} z_{3}, & & -i\left(\lambda_{1}-\lambda_{3}\right)^{1 / 2} z_{3}, \\
\eta_{3}= & z_{4}+i z_{5}, & \eta_{3}^{\prime}=z_{4}-i z_{5} .
\end{array}
$$

Then the smallest projective space $V$ containing the cone $\Lambda$ is given by

$$
V=\left\{\eta \in \mathbf{C P}^{5}: \eta_{2}^{\prime}=0, \eta_{3}=0\right\} .
$$

On $V$, we use $\left[\eta_{1}: \eta_{1}^{\prime}: \eta_{2}: \eta_{3}^{\prime}\right]$ as coordinate. Note that a point in $V$ has $\eta_{3}^{\prime}=0$ if and only if this point is on the plane of the conic $L$. Therefore, the open set $\Lambda \backslash L$ on the cone $\Lambda$ is contained in $W=\left\{\eta \in V: \eta_{3}^{\prime} \neq 0\right\}$. On $W$, we define $w$-coordinate:

$$
w=\left(w_{1}, w_{2}, w_{3}\right)=\left(\frac{\eta_{1}}{\eta_{3}^{\prime}}, \frac{\eta_{1}^{\prime}}{\eta_{3}^{\prime}}, \frac{\eta_{2}}{\eta_{3}^{\prime}}\right) .
$$

The center of this coordinate is exactly the vertex of the cone $\Lambda$. Now we blow up the $w$-coordinate at the center, then the proper transform of the cone is the Hirzebruch surface $F_{2}$ [16]. This copy of the Hirzebruch surface with the conic $L$ removed is denoted by $X_{1}$. The infinity section is denoted by $E_{\infty}$. Note that $X_{1} \backslash E_{\infty}$ is diffeomorphic to $\Lambda \backslash\left(\left\{y_{0}\right\} \cup L\right)$, which is diffeomorphic to $A^{0} \cong$ $R P^{3} \times I^{0}$. In terms of the $w$-coordinate, there is a standard way to write coordinates on the blowing-up of $W$ (p. 184 in [16]). It gives the standard coordinate on $X_{1}$. Therefore, given any real $\alpha$-conic with real parameter in $A^{0}$, one can first work out the intersection point on this real $\alpha$-conic and the cone $\Lambda$ in terms of the $\eta$-coordinate. After changing from $\eta$ to the $w$-coordinate, we change to the standard coordinate on $X_{1}$. This is a diffeomorphism from $A^{0}$ to $X_{1} \backslash E_{\infty}$. This map can be explicitly written down. Then one can check that the rational curve $E_{\infty}$ on $X_{1}$ parametrizes the real $\alpha$-conics with $t=-\lambda_{4}$. Moreover, the metric $g$ on $A^{0}$ can be extended from $X_{1} \backslash E_{\infty}$ to $X_{1}$ in the standard coordinate. As all the above coordinate changes are simple and standard, we do not intend to demonstrate the checking explicitly. 
Dually, to resolve the coordinate singularity at $t=-\lambda_{1}$, one can find two and only two real $\alpha$-conics passing through $y_{0}$ and $J\left(y_{0}\right)$ such that they jointed to $x_{0}$ to define simple cones in $Q_{0} \cap Q_{\infty}$. Picking up any one of them, we have a copy of $\mathbf{C P}^{3}$ containing this cone. We blow up this copy of $\mathbf{C P}^{3}$ at the vertex of the cone, then the proper transform of the cone is a Hirzebruch surface $F_{2}$. When the real $\alpha$-conic on $F_{2}$ is removed, the resultant space $X_{2}$ can be proved to be the parameter space of all real $\alpha$-conics in $Q_{0} \cap Q_{\infty}$ missing $y_{0}$ and $J\left(y_{0}\right)$. The infinity section in $X_{2}$ is the parameter space of all real $\alpha$-conics through $x_{0}$ and $J\left(x_{0}\right)$. With the infinity section on $X_{2}$ removed, the resultant space is diffeomorphic to $A^{0}$. This diffeomorphism carries the metric from $A^{0}$ to $X_{2} \backslash E_{\infty}$ and then the metric is extended over $X_{2}$.

As $X_{1}$ and $X_{2}$ contain an open submanifold $A^{0}$, one can glue $X_{1}$ and $X_{2}$ together along $A^{0}$ to obtain a compact smooth manifold $X$. One may regard $X_{1}$ and $X_{2}$ as two submanifolds in $X$ such that $X=X_{1} \cup X_{2}$ and $A^{0}=X_{1} \cap$ $X_{2}$. Then the extension of the metric $g$ from $A^{0}$ to $X_{1}$ and $X_{2}$ is an extension from $A^{0}$ to $X$. This is a self-dual metric on $X$. Its conformal class is denoted by $C_{\lambda}$. Since the topologies of $X_{1}, X_{2}$, and $A^{0}$ are known, one can use Van Kampen's Theorem and the Mayer-Vietoris sequence [32] to find that $X$ is a simply connected manifold with second betti number $b_{2}(Z)=2$. Using the self-duality, one can check that the intersection form is positive definite. Then one may appeal to Freedman's Theorem [14] to see that $X$ is homeomorphic to $\mathbf{C P}^{2} \# \mathbf{C} \mathbf{P}^{2}$.

Remark 4.1. To construct the conformal class $C_{\lambda}$ and $X$, we have made several choices. First of all, we choose to work on $\alpha$-conics instead of $\beta$-conics. Note that a coordinate change on $\mathbf{C P}^{5}$ can interchange our notion of $\alpha$-conics and $\beta$-conics. Then we choose one of the two exceptional real $\alpha$-conics through $x_{0}$ and $J\left(x_{0}\right)$. This conic is joined to $y_{0}$ instead of $J\left(y_{0}\right)$ to determine a cone. Using this cone, we resolve the coordinate singularity at $t=-\lambda_{4}$. We have to go through the same sort of choices to resolve the singularities at $t=-\lambda_{1}$. However, if one happens to make a different decision in the above choices, one constructs the same metric $g$ as ours because the resultant metric on $X$ agrees with our metric $g$ on the dense open set $A^{0}$ in the compact space $X$.

\section{The scalar curvature}

According to the work of Aubin and Schoen [6], [30], within the conformal class of any Riemannian metric on a compact manifold with dimension at least 3 , there is a metric with constant scalar curvature. We shall prove that $C_{\lambda}$ on $X$ cannot contain a metric with constant zero scalar curvature or negative scalar curvature. 
Proposition 5.1. If a compact connected 4-manifold $X$ has a self-dual metric with zero scalar curvature, then $\tau \geqslant \frac{2}{3} \chi$, where $\tau$ is the signature and $\chi$ is the Euler characteristic of the manifold $X$.

Proof. On an oriented 4-manifold, the curvature tensor of a metric has four $S O$ (4)-irreducible components: the scalar curvature $s$, the tracefree part of the Ricci tensor $B$, the self-dual Weyl tensor $W_{+}$, and the anti-self-dual Weyl tensor $W_{-}$. Using the Chern-Weyl formula, one can express the first Pontrjagin number $p_{1}$ and the Euler characteristic by

$$
\begin{gathered}
3 \tau=p_{1}=\frac{1}{4 \pi^{2}} \int_{X}\left|W_{+}\right|^{2}-\left|W_{-}\right|^{2}, \\
X=\frac{1}{8 \pi^{2}} \int_{X} \frac{s^{2}}{24}-2|B|^{2}+\left|W_{+}\right|^{2}+\left|W_{-}\right|^{2}
\end{gathered}
$$

(see [8], [17]). Under our assumption that $s \equiv 0$ and $W_{-} \equiv 0$, the result is obvious.

Proposition 5.2. Let $Z$ be the twistor space of a compact connected self-dual manifold $X$. If the scalar curvature is negative, then $H^{0}\left(Z, \mathcal{O}\left(\mathbf{K}^{-1 / 2}\right)\right)=0$.

Proof. Recall that the complexified bundle of anti-self-dual 2 -forms is isomorphic to the symmetric tensor product of the spin $-\frac{1}{2}$-bundle $-V_{-}^{2}$. The Levi-Civita connection on $X$ induces a connection:

$$
\nabla: C^{\infty}\left(\mathbf{V}_{-}^{2}\right) \rightarrow C^{\infty}\left(\mathbf{V}_{-}^{2} \otimes \Lambda_{\mathbf{C}}^{1}\right)
$$

The bundle $\mathbf{V}_{-}^{2} \otimes \Lambda_{\mathrm{C}}^{1}$ has an $S O(4)$-irreducible component $\mathbf{V}_{-}^{3} \otimes \mathbf{V}_{+}$. Then the connection defines an operator:

$$
D: C^{\infty}\left(\mathbf{V}_{-}^{2}\right) \rightarrow C^{\infty}\left(\mathbf{V}_{-}^{3} \otimes \mathbf{V}_{+}\right) \text {. }
$$

Let $\nabla^{*}$ and $D^{*}$ be the formal adjoints of the above differential operators and $s$ the scalar curvature. By self-duality, one obtains a Weitzenböch formula

$$
D * D=\nabla^{*} \nabla-\text { as, }
$$

where $a$ is a positive constant determined by the weight of the representation of $S O(4)$ which defines the bundle $\mathrm{V}_{-}^{2}$. When $s$ is negative and $X$ is compact, this formula shows that if $\phi$ is a section in $\mathbf{V}_{-}^{2}$ annihilated by $D$, then $\phi$ is the zero section.

On the other hand, we have seen in $\$ 2$ that a holomorphic section $\phi^{V}$ of the bundle $K^{-1 / 2}$ on the twistor space defines a section $\phi$ of the bundle $\mathbf{V}_{-}^{2}$. In fact, one may spell out the definition of the complex structure on the twistor space and the tautologically constructed bundle $\mathbf{K}^{-1 / 2}$ to see that the holomorphicitiy of $\phi^{V}$ implies that $\phi$ is annihilated by the operator $D$ [20]. Therefore, $K^{-1 / 2}$ does not have any holomorphic sections when $s$ is negative. 
Proposition 5.3. Let $Z$ be the twistor space of a connected self-dual manifold $X$. There is a vector bundle $\mathbf{E}$ with self-dual connection on $X$ such that it is pulled back to be the holomorphic bundle of 2-jets of $\mathbf{K}^{-1 / 2}$ on $Z, J_{2}\left(\mathbf{K}^{-1 / 2}\right)$. Moreover, a real holomorphic section of $J_{2}\left(\mathbf{K}^{-1 / 2}\right)$ is the pull-back of a covariant constant section of $\mathbf{E}$.

Proof. This proposition is within the framework of the so-called Ward correspondence initiated by Ward and fully developed in [3]. According to Theorem 5.2 in [3], the existence of the bundle $\mathbf{E}$ with the accompanied connection is ensured by (i) the holomorphicity of $J_{2}\left(\mathrm{~K}^{-1 / 2}\right)$, (ii) the reality of $J_{2}\left(\mathbf{K}^{-1 / 2}\right)$, and (iii) the triviality of $J_{2}\left(K^{-1 / 2}\right)$ along every real twistor line. In this occasion, the requirements (i) and (ii) are obviously satisfied; we have to prove (iii). After we prove this, the second statement of the proposition follows from the reality because, according to the Ward correspondence, the $\bar{\partial}$ operator on $J_{2}\left(\mathbf{K}^{-1 / 2}\right)$ is the $(0,1)$-part of the pull-back of the connection on $\mathbf{E}$.

Let $L$ be any real twistor line and $z$ any point on $L . S^{n} N^{*}$ denotes the $n$th symmetric product of the conormal bundle of $L$ in the twistor space, $\mathfrak{s}$ the ideal sheaf of $L$. For any nonnegative integer $n$, we define $\mathcal{O}_{L}^{(n)}=\mathcal{O} / \mathfrak{J}^{n+1}$. These sheaves $\mathcal{O}_{L}^{(n)}$ are supported by $L$. We use $\mathcal{O}_{L}^{(n)}(2)$ to denote the tensor product of $\mathcal{O}_{L}^{(n)}$ and the sheaf of germs of sections of $\mathbf{K}^{-1 / 2}$. On $L$, we have the exact sequence of sheaves:

$$
0 \rightarrow \mathcal{O}_{L}\left(S^{n} \mathbf{N}^{*} \otimes \mathbf{K}_{\mid L}^{-1 / 2}\right) \rightarrow \mathcal{O}_{L}^{(n)}(2) \rightarrow \mathcal{O}_{L}^{(n-1)}(2) \rightarrow 0 .
$$

Since $\mathbf{N}$ is isomorphic to $\mathbf{H} \oplus \mathbf{H}$ and $\mathbf{K}_{\mid L}^{-1 / 2}$ is isomorphic to $\mathbf{H}^{2}$, when $n=1$ and 2 , we have the induced exact sequence:

$$
\begin{aligned}
& 0 \rightarrow H^{0}\left(L, \mathbf{N}^{*} \otimes \mathbf{K}_{L}^{-1 / 2}\right) \stackrel{j}{\longrightarrow} H^{0}\left(L, \mathcal{O}_{L}^{(1)}(2)\right) \stackrel{\pi_{1}}{\longrightarrow} H^{0}\left(L, \mathcal{O}_{L}(2)\right) \rightarrow 0, \\
& 0 \rightarrow H^{0}\left(L, S^{2} \mathbf{N}^{*} \otimes \mathbf{K}_{L}^{-1 / 2}\right) \stackrel{j}{\longrightarrow} H^{0}\left(L, \mathcal{O}_{L}^{(2)}(2)\right) \stackrel{\pi_{2}}{\longrightarrow} H^{0}\left(L, \mathcal{O}_{L}^{(1)}(2)\right) \rightarrow 0 .
\end{aligned}
$$

Moreover, we can see that $h^{0}\left(L, \mathcal{O}_{L}^{(2)}(2)\right)=10$. Every element in $H^{0}\left(L, \mathcal{O}_{L}^{(2)}(2)\right)$ is evaluated at the point $z$. This evaluation defines a linear map from a 10- $\operatorname{dim}_{C}$ space to a $10-\operatorname{dim}_{C}$ space:

$$
e: H^{0}\left(L, \mathcal{O}_{L}^{(2)}(2)\right) \rightarrow J_{1}\left(\mathbf{K}^{-1 / 2}\right) z
$$

We claim that the evaluation map $e$ is an isomorphism. Let $s$ be a real element in $H^{0}\left(L, \mathcal{O}_{L}^{(2)}(2)\right)$ such that its second jet vanishes at $z$. As every section of $\mathbf{K}^{-1 / 2}$ over $L$ is a homogeneous polynomial of degree 2 with respect to a homogeneous coordinate on $L, s$ vanishes identically in the tangential direction of $L$. Therefore $\pi_{1} \circ \pi_{2}(s)=0$. Then $\pi_{2}(s)$ is in the image of $j_{1}$ and hence is regarded as an element in $H^{0}\left(L, \mathbf{N}^{*} \otimes \mathrm{K}^{-1 / 2}\right)$. This vector space is in 
turn identified to the tangent space of the complexified manifold $X^{c}$ at the parameter $x$ of $L$. According to Penrose's programme the cone $\Omega_{x}$ containing all sections of $\mathbf{N}$ vanishing somewhere on $L$ is the complex bilinear extension of the positive definite conformal structure on $X$. As the evaluation of $s$ at $z$ is zero, $\pi_{2}(s)$ is in the cone $\Omega_{x}$. But it is real; then $\pi_{2}(s)=0$. Therefore, $s$ is a constant section defined by an element in $H^{0}\left(L, S^{2} \mathbf{N}^{*} \otimes \mathrm{K}^{-1 / 2}\right)$. Then the evaluation of $s$ at $z$ is equal to zero only if $s$ is identically zero. Therefore, the evaluation map has no real kernel. As the real space $H^{0}\left(L, \mathcal{O}_{L}^{(2)}(2)\right)$ is spanned by real sections, the evaluation map is an isomorphism.

Since this is true for every point on $L$, the bundle $J_{2}\left(\mathrm{~K}^{-1 / 2}\right)$ restricted onto any real twistor line $L$ is trivialized by elements in $H^{0}\left(L, \mathcal{O}_{L}^{(2)}(2)\right)$.

As our manifold $X$ is homeomorphic to $\mathbf{C P}^{2} \# \mathbf{C P}^{2}$, Proposition 5.1 shows that the conformal classes $C_{\lambda}$ cannot contain metrics with zero scalar curvature. The manifold $X$ contains $\dot{A}$, the parameter space of all real $\alpha$-conics missing the singular points of $Q_{0} \cap Q_{\infty}$. The abstractly defined twistor space $Z$ over $X$ contains an open submanifold $Z^{0}$ which is the twistor space over $A^{0}$ and is holomorphically identified as an open submanifold in the variety $Q_{0} \cap Q_{\infty}$. Using the adjunction formula on $\mathbf{C P}^{5}$ and then on $Q_{0}$, one can see that the bundle $\mathrm{K}^{-1 / 2}$ on $Z^{0}$ is the restriction of the hyperplane bundle on $\mathbf{C P}^{5}$. As there are real hyperplanes in $\mathbf{C P}^{5}$, there are nontrivial real sections of $\mathbf{K}^{-1 / 2}$ over $Z^{0}$. Let $s$ be one of them. Its 2 -jet $j_{2}(s)$ is a nontrivial real holomorphic section $J_{2}\left(\mathrm{~K}^{-1 / 2}\right)$ over $Z^{0}$. According to the last proposition, $j_{2}(s)$ is the pull-back of a covariant constant section $\phi$ of the bundle $\mathbf{E}$ restricted on $A^{0} . \phi$ is parallely transported to the complement of $A^{0}$ in $X$. Since parallel transportation depends on initial data smoothly, one can check that the parallel transportation of $\phi$ from $A^{0}$ extends $\phi$ to be a covariant constant section of $\mathbf{E}$ over $X$. Now the pull-back of $\phi$ to the twistor space is a holomorphic section of $J_{2}\left(\mathrm{~K}^{-1 / 2}\right)$ over $Z$. This gives an extension of $s$ from $Z^{0}$ to $Z$. Therefore, $K^{-1 / 2}$ has nontrivial section. From Proposition 5.2, we see that the conformal classes $C_{\lambda}$ cannot contain a metric with negative scalar curvature. Then the conclusion is that the conformal classes $C_{\lambda}$ must contain a metric with constant positive scalar curvature. From Theorem 3.4, we see that $X$ is diffeomorphic to $\mathbf{C P}^{2} \# \mathbf{C P}^{2}$.

Theorem 5.4. There is a one-parameter family of self-dual conformal classes $C_{\lambda}, 2>\lambda>\frac{3}{2}$, on $\mathbf{C P}^{2} \# \mathbf{C P}^{2}$. Each of these conformal classes contains a metric with constant positive scalar curvature.

On the other hand, the twistor space $Z$ associated to any compact simply-connected self-dual manifold $X$ with positive scalar curvature is described by Theorem 3.5 with an appropriate $\lambda$. If necessary, a sign change on the base of $H^{0}\left(Z, \mathcal{O}\left(\mathbf{K}^{-1 / 2}\right)\right)$ ensures us that the associated map of $\mathbf{K}^{-1 / 2}$ sends 
real twistor lines to real $\alpha$-conics. If we follow the procedure in the last section to produce a metric $g$, then the metric $g^{\prime}$ on $X$ and $g$ are conformally equivalent on a dense open set $\mathbf{R P}^{3} \times I^{0}$ if we identify $X$ to $\mathbf{C P}^{2} \# \mathbf{C P}^{2}$. Then $g$ and $g^{\prime}$ are conformally equivalent to $\mathbf{C P}^{2} \# \mathbf{C P}^{2}$, i.e.,

Theorem 5.5. Any compact simply-connected self-dual manifold with positive scalar curvature and signature 2 is conformally equivalent to $\mathbf{C P}^{2} \# \mathbf{C P}{ }^{2}$ with a $C_{\lambda}$ for an appropriate $\lambda$ in the interval $\left(\frac{3}{2}, 2\right)$.

Finally, we want to determine the identity component of the group of conformal transformation with respect to the conformal class $C_{\lambda}$. From the conformal invariance of the holomorphic structure on the twistor space, one sees that an orientation preserving conformal diffeomorphism on $\mathbf{C P}^{2} \# \mathbf{C P}^{2}$ induces a biholomorphism on the twistor space $Z$ onto itself [3]. This biholomorphic map commutes with the real structure and hence induces a real linear automorphism $H^{0}\left(Z, \mathcal{O}\left(\mathrm{K}^{-1 / 2}\right)\right)$. This automorphism defines a real (linear) map $F$ from $C^{5}$ onto itself. $F$ is also an automorphism on $\Phi(Z)$, where $\Phi$ is the associated map of the system $\left|-\frac{1}{2} K\right|$. As $\Phi$ is an embedding modulo the set (c) of points on exceptional curves, if the induced map $F$ is an identity, the automorphism on the twistor space leaves every point on the dense set $Z \backslash$ \& fixed and hence must be an identity map on the twistor space. Then the conformal diffeomorphism is the identity map. Therefore, a nontrivial conformal diffeomorphism induces a nontrivial automorphism $F$ on $\Phi(Z)$. Since $\Phi(Z)$ is the intersection of the two quadrics given by (3.6), using the reality of $F$, one can easily prove that the action of $F$ on $\mathbf{C}^{6}$ in the coordinates $\left(z_{0}, \cdots, z_{5}\right)$ is simply rotating the $\left(z_{0}, z_{1}\right)$-plane and the $\left(z_{4}, z_{5}\right)$-plane by real $S O$ (2)-actions. Translated back onto $\mathbf{C P}^{2} \# \mathbf{C P}{ }^{2}$, on the $(x, y, \omega, t)$-coordinate, these rotations are exactly the rotation of the $(x, y)$-plane and the translation of the angular coordinate $\omega$. From this local expression, we actually see that this torus $S^{1} \times S^{1}$ action on $\mathbf{C P}^{2} \# \mathbf{C P}^{2}$ is in fact in the identity component of the group of isometry with respect to the metric $g$. Now we have

Theorem 5.6. The identity component of the group of conformal transformation with respect to the conformal structure $C_{\lambda}$ on $\mathbf{C P}^{2} \# \mathbf{C P}^{2}$ is a twodimensional torus.

\section{Further developments}

The natural questions to ask are whether there is self-dual conformal class on $3 \mathbf{C P}^{2} \equiv \mathbf{C P}^{2} \# \mathbf{C P}^{2} \# \mathbf{C P}^{2}$, whether there is a metric in this conformal class with positive scalar curvature, whether we can find out the complete family of a compact, simply-connected self-dual manifold $X$ with positive scalar curva- 
ture and signature 3 . It is very hopeful that we have affirmative answers. In fact, concerning the third question, one may use the bundle $D_{111}$ as we did in $\S 3$ to see that the manifold $X$ is diffeomorphic of $3 \mathbf{C P}^{3}$. In view of Hitchin's computation [21], we expect the singular model of the twistor space over $3 \mathbf{C P}^{2}$ can be given by a double covering of $\mathbf{C P} \mathbf{P}^{3}$ branched over a singular quartic.

However, this generalization has a natural obstruction, namely, the sign of $c_{1}^{3}(Z)$. In general, $c_{1}^{3}(Z)=2 \chi-3 \tau$. One may have noticed that our method in $\$ 2$ of searching for singular models of twistor spaces depends heavily, if not completely, on the abundance of effective divisors in the twistor space. This is successful if $\tau$ is less than 4 because $c_{1}^{3}(Z)$ is the leading coefficient of the Riemann-Roch formula. Therefore, it is fair to say that our approach is subject to the constraint that $\tau<\frac{2}{3} \chi$.

It is interesting to see that the constraint on the sign of $c_{1}^{3}(Z)$ occur in various occasions. It is a long time since Hitchin [19] proved that compact Einstein 4-folds have to satisfy the condition that $|\tau| \leqslant \frac{2}{3} \chi$. The proof is simply putting $B$ to be zero in (5.1) and (5.2), which are well understood today. Using these equations, we proved that a compact self-dual manifold with zero scalar curvature has to have $\tau \geqslant \frac{2}{3} \chi$. At this point, one might start guessing at the existence of self-dual metrics on $\tau \mathbf{C P}^{2}$ or even at the sign of the scalar curvature of a self-dual metric, but we would rather ask another question: According to Corollary 2.8, the conformal class of the Fubini-Study metric is the unique one containing a metric with positive scalar curvature on $\mathbf{C P}^{2}$. Proposition 5.1 shows that there is no metric on $\mathbf{C P}^{2}$ which is self-dual with zero scalar curvature, but

Is there any self-dual metric with (constant) negative scalar curvature on $\mathbf{C P}^{2}$ ?

Finally, we should point out that the work in $\$ 3$ has side-product. Before we drew the conclusion in Theorem 3.5, we studied the real structure on the intersection of two quadrics:

$$
\begin{aligned}
& Q_{0}=\left\{w \in \mathbf{C P}^{5}: a w_{0}^{2}+a w_{2}^{2}+b w_{2}^{2}+b w_{3}^{2}+c w_{4}^{2}+d w_{5}^{2}=0\right\} \\
& Q_{\infty}=\left\{w \in \mathbf{C P}^{5}: w_{0}^{2}+w_{1}^{2}+w_{2}^{2}+w_{3}^{2}+w_{4}^{2}+w_{5}^{2}=0\right\} .
\end{aligned}
$$

Note that if the two rank 4 quadrics in the pencil are real as we use to have but the two rank 5 quadrics are conjugate to each other, then $Q_{0} \cap Q_{\infty}$ contains real points and, in fact, singular real conics. In this case, one can still normalize the coordinate so that the intersection is given by

$$
\begin{aligned}
& Q_{0}=\left\{z \in \mathbf{C P}^{5}: 2 z_{0}^{2}+2 z_{1}^{2}+\lambda z_{2}^{2}+\bar{\lambda} z_{3}^{2}+z_{4}^{2}+z_{5}^{2}=0\right\} \\
& Q_{\infty}=\left\{z \in \mathbf{C P}^{5}: z_{0}^{2}+z_{1}^{2}+z_{2}^{2}+z_{3}^{2}+z_{4}^{2}+z_{5}^{2}=0\right\}
\end{aligned}
$$


with $\operatorname{Im} \lambda>0$ and the real structure $J$ is given by

$$
J\left(z_{0}, \cdots, z_{5}\right)=\left(\bar{z}_{0}, \bar{z}_{1}, \bar{z}_{3}, \bar{z}_{2},-\bar{z}_{4},-\bar{z}_{5}\right) .
$$

Then using the setting in $\$ 4$, one can also work out a self-dual metric on a noncompact manifold. The noncompactness is due to the fact that a nonsingular real $\alpha$-conic may deform to a singular real $\alpha$-conic in $Q_{0} \cap Q_{\infty}$. This manifold is expected to be the connected sum of two unit open discs, i.e. the cylinder $S^{3} \times \mathbf{R}$. The more interesting questions are whether there is a complete metric in this self-dual conformal class and, if it exists, whether its scalar curvature is negative.

\section{Appendix}

Moduli space of self-dual connections. An example. It is well known that there are several approaches to construct instantons, i.e. self-dual connections on a principal $S U(2)$-bundle, over the Euclidean 4-sphere: a $t^{\prime}$ Hoopf construction described by Atiyah and Ward [5], the Serre construction given by Hartshorne [17], the ADHM construction given by Atiyah, Drinfeld, Hitchin, and Manin [2] and a monad construction given by Barth [7]. The first approach is a differential geometric computation on $S^{4}$. The second and third approaches are to translate the problem to the construction of a special class of holomorphic rank 2 bundles on $\mathbf{C P}^{3}$, the twistor space of the sphere. The fourth is to construct rank 2 bundles on $\mathbf{C P}^{2}$, a hyperplane in the twistor space. The translation is given by the Ward correspendence:

Theorem 7.1 ([3], [5]). Let $E$ be an $S U(2)$-bundle on a self-dual manifold $X$ with a self-dual connection and $F$ the pulled back bundle of $E$ onto the twistor space. Then (i) $F$ is a holomorphic bundle, (ii) $F$ has a quaternionic structure, and (iii) $F$ is holomorphically trivial along every real twistor line. Conversely, any rank 2 bundle on the twistor space with the above three properties is the pull-back of an $S U(2)-b u n d l e$ on $X$ with a self-dual connection.

We shall make use of this Ward correspondence and essentially a Serre construction to find self-dual $S O(3)$-connections on $X=\mathrm{CP}^{2} \# \mathrm{CP}^{2}$. On this manifold, we always fix a self-dual metric with positive scalar curvature. Associated to the twistor space over $X$ with the given conformal class $C_{\lambda}$ there is a pencil of quadrics $Q_{t}=Q_{0}+t Q_{\infty}$ in $\mathbf{C P}^{5}$, a real structure on $\mathbf{C P}^{5}$, and a map $\Phi: Z \rightarrow Q_{0} \cap Q_{\infty}$ such that $\Phi$ is an embedding modulo four curves and sending every real twistor line to a real conic. In this occasion, we choose a coordinate on $\mathbf{C P}^{5}$ such that the real twistor lines are sent to real $\beta$-conics 
(Remark 4.1). The planes of real $\beta$-conics are real planes contained in $Q_{t}$ for some real number $t$ in the interval $I$ in $\mathbf{R P}^{1}$ :

$$
I=(-\infty,-2] \cup[-1, \infty) \cup\{\infty\} .
$$

Now for any $t$ in the open interval $I^{0}$ in $I$ with the two endpoints $\{-1,-2\}$ removed, $Q_{t}$ is a smooth real quadric of dimension 4 . When we consider the smooth quadric as the Grassmannian of planes in $\mathbf{C}^{4}$, we have the universal subbundle $U_{t}$ over $Q_{t}$ [16]. The projectivization of the dual of $U_{t}$ is pulled back by the map $\Phi$ to be a holomorphic fiber bundle $\xi_{t}$ on $Z$. On the other hand, letting $\mathfrak{M}^{0}$ be the moduli space of irreducible self-dual connections on an $S O(3)$-bundle $\zeta$ on $X$ with second Stiefel-Whitney class $w_{2}(\zeta)=w_{2}(X)$ and first Pontrjagin number $p_{1}(\zeta)=2$, we shall prove the following:

Theorem 7.2. There is a bijective correspondence

$$
I^{0} \leftrightarrow\left\{\xi_{t}: t \in I^{0}\right\} \leftrightarrow \mathfrak{M}^{0} .
$$

As far as the dimension of the moduli-space is concerned, this theorem is a consequence of Atiyah-Hitchin-Singer's computation [3]. In fact, Fintushel and Stern had exploited Donaldson's technique of studying the moduli of $S U(2)$ connections to study the moduli of $S O(3)$-connections. Part of their results restricted to our situation is the following:

Theorem 7.3 ([12], [13]). Let $\zeta$ be an SO(3)-bundle over $X=\mathbf{C P}^{2} \# \mathbf{C P}^{2}$. Assume that $p_{1}(\zeta)$ is equal to 2 and $w_{2}(\zeta)=w_{2}(X)$. Then

(i) the moduli space $\mathfrak{M}$ of all self-dual connections on $\zeta$ is compact,

(ii) there are two (equivalence classes of ) reducible self-dual connections on $\zeta$,

(iii) the (equivalence classes of) reducible self-dual connections on $\zeta$ are the two endpoints of a closed interval in $\mathfrak{M}$.

The virtue of our theorem is that we give an explicit construction of all irreducible connections and show that $\mathfrak{M}$ has only one connected component. Before we proceed to carry out the construction, it is worthwhile to remark that our moduli space is closely related to Newstead's moduli space of stable rank 2 bundles over a hyperelliptic curve [26].

To prove the theorem, we shall first show that $\xi_{t}$ and $\xi_{t^{\prime}}$ are inequivalent for $t \neq t^{\prime}$. Then each $\xi_{t}$ is shown to be uniquely determining an irreducible self-dual connection on $\zeta$. Finally, every irreducible self-dual connection is proved to be given by $\xi_{t}$.

Construction of self-dual connections. Given $t$ in $I^{0}$ and using the setting in \$4, we have the $\alpha$-planes $A(\sigma ; t), \sigma \in \mathbf{C P}^{3}$, in $Q_{t}$. As we use the Plücker embedding to identity $Q_{t}$ as the Grassmannian of lines in $\mathbf{C P}^{3}$, the $\alpha$-plane $A(\sigma ; t)$ consists of all lines in $\mathbf{C P}^{3}$ through the point $\sigma$. In other words, a nonzero vector $\vec{q}$ in $\mathbf{C}^{6}$ representing a point $q$ in $Q_{t}$ is in the kernel of the 
matrix $A(\sigma ; t)$ if and only if the line in $\mathbf{C P}^{3}$ represented by $q$ contains the point $\sigma$ in $\mathbf{C P}^{3}$. If one defines $A_{t, q} \equiv\left\{\sigma \in \mathbf{C P}^{3}: A(\sigma ; t) \vec{q}=0\right\}$, then it is easy to see that $A_{t, q}$ is the fiber of the projectivization of the universal bundle over $q$.

For any nonsingular conic $L$ in $Q_{0} \cap Q_{\infty}, A_{t}$ is trivial over $L$ except when the plane of $L$ is an $\alpha$-plane contained in $Q_{t}$. When $t \neq t^{\prime}$, we then see that $A_{t}$ is trivial on the nonsingular $\alpha$-conics $\alpha\left(\sigma ; t^{\prime}\right)$ on which $A_{t^{\prime}}$ is nontrivial. As the map $\Phi$ identifies some rational curves in $Z$ to the real $\alpha$-conics in $Q_{0} \cap Q_{\infty}$ and $\xi_{t}$ is the pull-back of the dual of $A_{t}$, then $\xi_{t}$ and $\xi_{t^{\prime}}$ on the twistor space are holomorphically inequivalent when $t \neq t^{\prime}$. This is the one-to-one correspondence $I^{0} \leftrightarrow\left\{\xi_{t}: t \in I^{0}\right\}$.

Moreover, the real twistor lines are mapped onto real $\beta$-conics; then each $\xi_{t}$ is holomorphically trivial along every real twistor line and the transition function of each $\xi_{t}$ defines a Čech cocycle on $X$ with value in $\operatorname{PGL}(2, \mathrm{C})$. This global observation can be refined by a local study.

Recall that the second Stiefel-Whitney class of the $S O(2)$-bundle $K^{-1 / 2}$ is equal to $w_{2}(X)=\alpha_{1}+\alpha_{2}$, where $\alpha_{1}$ and $\alpha_{2}$ is a pair of orthonormal generators of $H^{2}(X, Z)$. Therefore, the bundle $K^{-1 / 2}$ is not the second power of a holomorphic line bundle. However, if we choose to work on any small neighborhood on $X$, we can find a holomorphic line bundle $K^{-1 / 4}$ on the inverse image of this open set on the twistor space such that $\left(K^{-1 / 4}\right)^{2}=K^{-1 / 2}$. The restriction of $K^{1 / 4}$ on any real twistor line $L$ has degree -1 . It has an induced quaternionic structure defined by the antipodal map on $L \cong \mathbf{C P}^{1}$. On the other hand, the universal subbundle $U_{t}$ on $Q_{t}$ inherits a real structure from the induced real structure on the trivial rank 4 bundle, $Q_{t} \times \mathrm{C}^{4}$, given by

$$
\left(\sigma_{0}, \sigma_{1}, \sigma_{2}, \sigma_{3}\right) \mapsto\left(\bar{\sigma}_{2},-\bar{\sigma}_{3}, \bar{\sigma}_{0},-\bar{\sigma}_{1}\right)
$$

(cf. (4.1)). Therefore, the bundle $F_{t} \equiv \Phi^{*}\left(U_{t}^{*}\right) \otimes K^{1 / 4}$ on the twistor space has a quaternionic structure wherever this is defined.

As the bundle $\Phi^{*}\left(\bigwedge^{2} U_{t}\right)$ is simply the pull-back of the universal line bundle on $\mathrm{CP}^{5}$ and the map $\Phi$ is the associated map of the bundle $K^{-1 / 2}$, the first Chern class of $F_{t}$ vanishes. Then the transition function of $F_{t}$ takes value in $S L(2, \mathrm{C})$ with respect to a good covering. $S L(2, \mathrm{C})$ has two real forms [18]. One is $S U(2)$, the real form determined by a quaternionic structure. Another is $S L(2, \mathbf{R})$, the real form determined by a real structure. Now $F_{t}$ has quaternionic structure and hence is a $S U(2)$-bundle. Moreover, the projectivization of $F_{t}$ is exactly the bundle $\xi_{t}$. It shows that $\xi_{t}$ is a $P S U(2)$-bundle. The triviality of $\xi_{t}$ on each real twistor line implies that $F_{t}$ is a sum of two line bundles with equal degree on each real twistor line. As the first Chern class of $F_{t}$ vanishes, $F_{t}$ is trivial along each real twistor line. Then by the Ward correspondence, $F_{t}$ 
is the pull-back of an $S U(2)$-bundle $E_{t}$ on an open set of $X$ with a self-dual connection. The obstruction for $E_{t}$ globally defined on $X$ is $w_{2}(X)$. However, the adjoint bundle of $E_{t}, \zeta_{t}$, is a well-defined $S O(3)$-bundle on $X$. The isomorphism of the Lie algebra of $S U(2)$ and $S O(3)$ carries the self-dual connection on $E_{t}$ over each small open set to a self-dual connection on $\zeta_{t}$ over $X$.

The second Stiefel-Whitney class of $\zeta_{t}$ is exactly the obstruction for $\zeta_{t}$ to be the adjoint bundle of a globally defined $S U(2)$-bundle, therefore $w_{2}\left(\zeta_{t}\right)=$ $w_{2}(X)$. Moreover, the Chern classes of the universal subbundle over a quadratic is known [16]. Then it is easy to show that $p_{1}\left(\zeta_{t}\right)=2 e(X)-p_{1}(X)$, where $e(X)$ and $p_{1}(X)$ are the Euler class and the first Pontrjagin class of the manifold respectively. Hence $p_{1}\left(\zeta_{t}\right)[X]=2$. Since any two $S O(3)$-bundles over a 4-fold with the same second Stiefel-Whitney classes and the same first Pontrjagin classes are isomorphic [10], all $\zeta_{t}$, with $t$ in $I^{0}$, are isomorphic to the bundle $\zeta$. As $\Phi^{*} U_{t}$ is holomorphically inequivalent to $\Phi^{*} U_{t^{\prime}}$ when $t \neq t^{\prime}$, Ward correspondence also shows that $\zeta_{t}$ and $\zeta_{t^{\prime}}$ are distinguished by their inequivalent self-dual connections. Therefore, we obtain an open interval $I^{0}$ in the moduli space. According to the theorem of Fintushel and Stern, reducible self-dual connections are not in the interior of an interval, hence all the self-dual connections determined by the $\xi_{t}$ 's are irreducible. So we have a one-to-one map $\left\{\xi_{t}: t \in I^{0}\right\} \rightarrow \mathfrak{M}^{0}$.

Remark 7.4. It can be checked that the bundle $U_{t}$ has a quaternionic structure when $t$ is in $(-2,-\lambda) \cup\left(-\frac{3}{2},-1\right)$. Then $\Phi^{*} U_{t}^{*} \otimes K^{1 / 4}$ has a real structure and hence $\xi_{t}=P\left(\Phi^{*} U_{t}^{*}\right)$ is a $P S L(2, \mathbf{R})$-bundle. Then the Ward correspondence gives us a family of $S O(2,1)$ self-dual connections, the objects we do not pursue here.

Completeness of the parametrization. Given the bundle $\zeta$ with prescribed topology, one sees that locally it is the adjoint bundle of an $S U(2)$-bundle $E$. Let $F$ be the pull-back of $E$ onto the twistor space. Then formally,

$$
c_{1}(F)=0 \text { and } c_{2}(F)=\frac{1}{4}\left(\alpha_{1}^{2}+\alpha_{2}^{2}\right) .
$$

Given any irreducible self-dual connection on $\zeta$, according to the construction in the Ward correspondence, the $(0,1)$-part of the pull-back connection on $F$ defines a holomorphic structure. Then the bundle $U^{*} \equiv F \otimes K^{-1 / 4}$ is a globally defined holomorphic rank 2 bundle on the twistor space. It is globally defined because $w_{2}(\xi)=w_{2}\left(K^{-1 / 2}\right)$. It has

$$
c_{1}\left(U^{*}\right)=\frac{1}{2} c_{1} \quad \text { and } \quad c_{2}\left(U^{*}\right)=\frac{1}{4}\left(\alpha_{1}^{2}+\alpha_{2}^{2}\right)+\frac{1}{16} c_{1}^{2} .
$$

Then the Riemann-Roch formula and Hitchin's vanishing theorem show that

$$
h^{0}\left(Z, \mathcal{O}\left(U^{*}\right)\right) \geqslant 4 \text {. }
$$


If there is a section of $U^{*}$ without zeros, this section defines a trivial subbundle of $U^{*}$. The first Chern class of the quotient line bundle of the trivial subbundle in $U^{*}$ is equal to the first Chern class of $K^{-1 / 2}$. But in $\S 1$ we have seen that the positivity of the scalar curvature implies that holomorphic line bundles on the twistor space over $X$ are uniquely determined by their first Chern classes. Therefore, the quotient bundle is exactly $K^{-1 / 2}$. Then $U^{*}$ is an extension of $K^{-1 / 2}$ by the trivial bundle. This extension has to be trivial because the obstruction of triviality is in $H^{1}\left(Z, \mathcal{O}\left(K^{1 / 2}\right)\right)$. This cohomology group vanishes because of Hitchin's vanishing theorem. Hence the locally defined bundle $F$ is given by

$$
F=U^{*} \otimes K^{1 / 4}=K^{-1 / 4} \oplus K^{1 / 4} .
$$

This splitting of $F$ implies the reducibility of the self-dual connection on $E$ [3]. Hence the self-dual connection on $\zeta$ is reducible. Therefore, under our assumption that the self-dual connection on $\zeta$ is irreducible, we can conclude that every section of $U^{*}$ has zeros. In fact, one can prove that every nontrivial real section of $U^{*}$ vanishes on a unique real twistor line.

On the other hand, the first Chern class of $U^{*}$ is equal to that of $K^{-1 / 2}$; then the determinant bundle of $U^{*}$ is holomorphically equivalent to $K^{-1 / 2}$. If $s_{0}$ and $s_{1}$ are sections of $U^{*}$, then $s_{0} \wedge s_{1}$ is a section of $K^{-1 / 2}$. As $s_{0} \wedge s_{1}$ vanishes identically only if $s_{0}(z)$ and $s_{1}(z)$ are linearly dependent over every point $z$ in the twistor space. This is equivalent to having a holomorphic function $f$ on the complement of the zeros of $s_{0}$ and $s_{1}$ in the twistor space such that $s_{0}=f s_{1}$. But the zero set of $s_{0}$ and $s_{1}$ has codimension 2 when $s_{0}$ and $s_{1}$ are real. Hartog's Theorem [16] shows that $f$ is extended to be a global holomorphic function on the twistor space and hence is a constant. Then $s_{0}$ and $s_{1}$ are linearly dependent in the vector space

$$
V=H^{0}\left(Z, \mathcal{O}\left(U^{*}\right)\right) \text {. }
$$

Therefore, we have a canonical injection:

$$
\wedge^{2} V \rightarrow H^{0}\left(Z, \mathcal{O}\left(K^{-1 / 2}\right)\right)
$$

Counting the dimensions, we see that $\operatorname{dim} V=4$ and the injection is an isomorphism. We shall use $V^{*}$ to denote the dual of $V$.

Let $\left\{s_{0}, \cdots, s_{3}\right\}$ be a real basis of $V$. Then

$$
\left\{s_{0} \wedge s_{1}, s_{2} \wedge s_{3}, s_{0} \wedge s_{2}, s_{3} \wedge s_{1}, s_{0} \wedge s_{3}, s_{1} \wedge s_{2}\right\}
$$

forms an ordered basis of $\Lambda^{2} V$. This choice determines a homogeneous coordinate on $P\left(\wedge^{2} V^{*}\right)$, the target space of the associated map $\Phi$ of $\left|-\frac{1}{2} K\right|$, and $\Phi$ sends real twistor lines to $\beta$-conics.

Let $z$ be any point in the twistor space; its ideal sheaf is denoted by $\mathfrak{\Im}_{z}$. 
Then we have an exact sequence

$$
0 \rightarrow H^{0}\left(Z, \mathfrak{\Im}_{z}\left(U^{*}\right)\right) \rightarrow H^{0}\left(Z, \mathcal{O}\left(U^{*}\right)\right) \rightarrow U_{z}^{*} \rightarrow \cdots .
$$

The restriction map at $z$ fails to be surjective only if $h^{0}\left(Z, \mathfrak{\Im}_{z}\left(U^{*}\right)\right)$ is at least equal to 3 . Then by the reality of the bundle $U^{*}, h^{0}\left(Z, \widetilde{\Im}_{J(z)}\left(U^{*}\right)\right)$ is also at least equal to 3, where $J(z)$ is the conjugate point of $z$. But $\operatorname{dim} V=4$; then $h^{0}\left(Z, \mathfrak{\Im}_{z, J(z)}\left(U^{*}\right)\right) \geqslant 2$. Since any section of $U^{*}$ vanishing at $z$ and $J(z)$ must vanish along the real twistor line $L$ through $z$, there are linearly independent real sections, say $s_{0}$ and $s_{1}$, which vanish along $L$. There are five-dimensional sections of $K^{-1 / 2}$ which vanish along a real twistor line. This is impossible. Therefore, the restriction map is surjective and we have the dual of an exact sequence:

$$
0 \rightarrow\left(U_{z}^{*}\right)^{*} \rightarrow V^{*} \rightarrow H^{0}\left(Z, \Im_{z}\left(U^{*}\right)\right) \rightarrow 0 .
$$

Then the image of the point $z$ via the associated map $\Phi$ is exactly representing the plane $U_{z}$ in $V^{*}$. Therefore, the Grassmannian of planes in $V^{*}$ is a nonsingular real member $Q_{t}$ of the pencil generated by $Q_{0}$ and $Q_{\infty}$. In the coordinate determined by a real basis $\left\{s_{0}, \cdots, s_{3}\right\}$, one can see that $Q_{t}$ is a real quadric with zero signature. Then in the original $z$-coordinate on $\mathbf{C P}^{5}$ and the given parametrization of the pencil of quadrics, one can check that $t$ is in the interval $I^{0}$. Now by the definition of universal subbundle, we have

$$
\left(\Phi^{*} U_{t}\right)_{z}=\left\{(\Phi(z), v) \in Q_{t} \times V^{*}: v \in \Phi(z)\right\}=\Phi(z)=U_{z} .
$$

Therefore, we have a canonical isomorphism $\Phi^{*} U_{t}^{*}=U^{*}$. The projectivization of $U^{*}$ is precisely $\xi_{t}$. It shows that the one-to-one map $\left\{\xi_{t}: t \in I^{0}\right\} \rightarrow \mathfrak{M}^{0}$ is surjective.

\section{References}

[1] M. F. Atiyah, K-theory and reality, Quart. J. Math. Oxford (2) 17 (1966) 367-386.

[2] M. F. Atiyah, V. Drinfeld, N. J. Hitchin \& Y. I. Manin, Construction of instantons, Phys. Lett. A 65 (1978) 185.

[3] M. F. Atiyah, N. J. Hitchin \& I. M. Singer, Self-duality in four dimensional Riemannian geometry, Proc. Roy. Soc. London Ser. A 362 (1978) 425-461.

[4] M. F. Atiyah \& I. M. Singer, The index of elliptic operator. III, Ann. of Math. (2) 87 (1968) 546-604.

[5] M. F. Atiyah \& R. S. Ward, Instantons and algebraic geometry, Comm. Math. Phys. 55 (1977) 117-124.

[6] T. Aubin, The Scalar curvature, Differential Geometry and Relativity (Cahen and Flato, eds.), Reider, 1976.

[7] W. Barth, Moduli of vector bundles on the projective plane, Invent. Math. 42 (1977) 63-91.

[8] A. Besse, Géometrie Riemanniene en dimension 4, Textes Mathématiques, No. 3, CEDIC, Fernard Nathan, Paris, 1981. 
[9] M. Demazure, Surfaces del Iel Pezzo. III, in Séminaire sur les Singularités des Surfaces, Lecture Notes in Math., No. 777, Springer, Berlin, 1980.

[10] A. Dold \& H. Whitney, Classification of oriented sphere bundles over a 4-complex, Ann. of Math. (2) 69 (1959) 667-677.

[11] S. K. Donaldson, An application of gauge theory to the topology of 4-manifolds, J. Differential Geometry 18 (1983) 269-316.

[12] R. Fintushel \& R. J. Stern, SO(3)-connections and the topologv of 4-manifolds, J. Differential Geometry 20 (1984) 523-539.

[13] D. S. Freed \& K. K. Uhlenbeck, Instantons and 4-manifolds, Math. Sci. Res. Inst. Series, Vol. 1, Springer, Berlin, 1984.

[14] M. Freedman, The topologv of four-dimensional manifolds, J. Differential Geometry 17 (1982) 357-454.

[15] T. Friedrich \& H. Kurke, Compact four-dimensional self-dual Einstein manifold with positive scalar curvature, Math. Nachr. 106 (1982) 271-299.

[16] P. Griffiths \& J. Harris, Principles of algebraic geometry, Wiley, New York, 1978.

[17] R. Hartshorne, Stable vector bundles and instantons, Comm. Math. Phys. 59 (1978) 1-15.

[18] S. Helgason, Differential geometry, Lie groups and symmetric spaces, Academic Press, London, 1978.

[19] N. J. Hitchin, Compact four-dimensional Einstein manifolds, J. Differential Geometry 9 (1974) 435-441.

[20] _ Linear field equations on self-dual spaces, Proc. Roy. Soc. London Ser. A 370 (1980) 173-191.

[21] __ Kählerian twistor spaces, Proc. London Math. Soc. (3) 43 (1981) 133-150.

[22] Self-duality, in A. Besse's Einstein manifold, Springer, Berlin, to appear.

[23] W. V. D. Hodge \& D. Pedoe, Methods of algebraic geometry, Vol. II, Cambridge University Press, Cambridge, 1947.

[24] J. Hurtubise, The intersection of two quadrics in $\mathbf{C P}^{5}$ as a twistor space, Annals of Global Analysis and Geometry, to appear.

[25] H. N. Kuiper, On conformally flat spaces in the large, Ann. of Math. (2) 50 (1949) 916-924.

[26] P. E. Newstead, Stable bundles of rank 2 and odd degree over a curve of genus 2, Topology 7 (1968) 205-215.

[27] T. H. Parker, Gauge theories on four-dimensional Riemannian manifold, Comm. Math. Phys. 85 (1982) 563-602.

[28] R. Penrose, Nonlinear gravitons and curved twistor theory, General Relativity and Gravitation 7 (1976) 31-52.

[29] F. Sakai, Anticanonical models of rational surfaces, Math. Ann. 269 (1984) 389-410.

[30] R. Schoen, Conformal deformation of a Riemannian metric to constant scalar curvature, J. Differential Geometry 20 (1984) 479-495.

[31] Y. T. Siu, A vanishing theorem for semipositive line bundles over non-Kühler manifolds, J. Differential Geometry 19 (1984) 431-452.

[32] E. H. Spanier, Algebraic topologv, McGraw-Hill, New York, 1966.

[33] K. Ueno, Classification theory of algebraic varieties and compact complex spaces, Lecture Notes in Math., No. 439, Springer, Berlin, 1975. 